\title{
LA CIRCUNSCRIPSIÓN DE PALICOUREA SUBGEN. HETEROPSYCHOTRIA (RUBIACEAE PALICOUREEAE)
}

\author{
A. L. BORHIDI \\ Instituto de Biología, Universidad de Pécs \\ H-7624 Pécs, Ifjúság útja 6, Hungría; E-mail: borhidi@gamma.ttk.pte.hu
}

(Received 23 November, 2016; Accepted 25 December, 2016)

The subgenus Heteropsychotria was detected and described by Julian Steyermark in the frame of the monographic series of the Botany of the Guyana Highland (Steyermark 1972) and repeatedly in a more illustrated version in the Flora of Venezuela (Steyermark 1974). Within the subgenus he distinguished 13 sections and 31 series. Three sections (Nrs 4, 7 and 11) of the 13 remained without names and descriptions. After the fundamental molecular taxonomic study of Nepokroeff et al. (1999) a publishing activity started producing a number of critical studies separating some sections as valid individual genera. The section Chytropsia has been included into the genus Margaritopsis Ch. Wright (Andersson 2001, Taylor 2005). The section Notopleura regained its generic status according to Bremekamp's original concept (Taylor 2001). The Mapouria section has been eliminated by the re-establishment of the Carapichea genus (Andersson 2002b, Taylor and Gereau 2013). It has been recently recognised, that the greater part of the species classified by Steyermark into the section Durifolia belongs to the expanded genus Coccochondra (Taylor 2011). The re-consideration of the taxonomic position of the slenderized subgenus Heteropsychotria was begun by C. M. Taylor et al. with the transfer of some Mesoamerican species as late as 2010, although their close relation to the genus Palicourea was emphasised by Nepokroeff et al. (1999). The next step is, that for the second edition of the Rubiáceas de México the Mexican species of Heteropsychotria were transferred into Palicourea (Borhidi 2011), while C. M. Taylor maintained the Mesoamerican ones within the frame of Psychotria (Taylor 2012). Recently C. M. Taylor started to revise the taxonomic position of the South American Heteropsychotria species with a new circumscription and re-description of the section Didymocarpae (Taylor 2014) by the distinction and description of the new section Tricephalium (Taylor 2015) separating from the rest of the section Cephaëlis and by the detailed elaboration of the section Nonatelia in a new concept with enlarged frame (Taylor and Hollowell 2016). For the remained parts of the Steyermark's system we suggest here the following reconsiderations. The section Heteropsychotria is enlarged by the inclusion of the anonymous section 4 of Steyermark. The greater part of the anonymous section 7 , which remained after the exclusion of the Carapi- 
chea species, is described as a new section Bracteiflorae with Palicourea caerulea as type. The section Nonatelia is reconsidered and reshaped in a considerably enlarged form by Taylor and Hollowell (2016), while the section Potaroenses remains unchanged. The Pseudocephälis section remains without the series Appunianae, which has been included into the section Tricephalium C. M. Taylor. The section Cephaëlis of Steyermark is divided into two sections. The species with terminal, mostly pedunculate inflorescences is separated under a new name Neocephaëlis Borhidi with Palicourea tomentosa (Aubl.) Borhidi, selected as the type species of the section. The other section includes the species of axillary, mostly sessile inflorescences, elevating the series Axillares of Steyermark on section rank and expanding it by the inclusion of the anonymous section 11, with Palicourea axillaris (Aubl.) Borhidi, selected as the type. For the rest of the eliminated Durifolia section a new one is created under the name Cordifoliae Borhidi, while the Oppositiflorae section also must be cancelled, because its type species is Ronabea latifolia Aubl. At last a new section Microphyllae Borhidi is established for the small-leaved xero-tolerant species of the Antilles with Palicourea orientensis Borhidi et Oviedo as type. The review includes more than 220 species.

Recently Taylor has made an attempt to eliminate the subgenus Heteropsychotria Steyerm. and identified it as a synonym of subgenus Palicourea. It cannot be accepted because the two subgenera differ from each other in important morphological and physiological characters.

The subgenus Palicourea is characterised by having brightly coloured odourless, mostly pedicellate and separated flowers with large corollas often curved tube and swollen base containing an appreciable quantity of nectar, protected by a ring of stiff hairs, namely an arranged complex of characters, which are adapted for hummingbird-pollination. The type species is Palicourea guianensis Aubl. In contrast, the subgenus Heteropsychotria is characterised by having generally green to white inflorescences with white greenish or pale yellow fragrance, smaller mostly sessile flowers with corollas of straight short tube without swollen base and inner hairy ring, but with different pubescence in/or above the middle of the inner face of the tube. These arranged set of characters are clearly adapted to the insect-pollination. The type species is Palicourea deflexa (Sw.) Borhidi. Taylor refers to a great variation in some separate characters between the two character complexes presented in the Flora of the Venezuelan Guayana (2004) without mentioning any concrete example. The reality is, that between the two character complexes there is no intermediate taxon.

After having replaced the subgenus Heteropsychotria Steyermark synonymised by Taylor, a new section has been introduced and created, the section Tricephalium (Müll. Arg.) C. M. Taylor, comprising 35 species and classified in Palicourea subgenus Montanae Taylor 1997. It is diagnosed by having "thick-textured leaves with well-developed intersecondary veins that are extensively reticulated and inflorescences with the flowers sessile in small to large heads that are enclosed by well-developed bracts and the fruits pass through a yellow to red immature stage". Additionally the section is also characterised by stipules that are shortly united around the stem to laminar, bilobed to emarginate, rounded, rounded and denticulate or erose in each side.

Really, the section Tricephalium is problematic in some respect. It is heterogeneous as a taxonomic unit, because it has not a standard set of characters, even not one character existing in all species classified here for the distinction of the whole group. The most common characteristic feature is the texture and venation of the leaves, a vegetative character that may be influenced rather easily by ecological conditions. Moreover, the major part of the species lives in montane valleys of the Andes of Colombia, Ecuador, Peru and Bolivia as isolated local endemics within similar montane habitats. It is unknown, how these charac- 
ters would react if the individuals of these species got into lowland situation (see: Clausen et al. (1948) on Achillea lanuginosa). It is to be mentioned that similar leaf texture venations occur in some Carapichea and Coccochondra species as well. Another weakness of the section is the superfluous selection of the typical species, the Palicourea triadica (Müll. Arg.) C. M. Taylor being a very scarcely collected and poorly known species is unable to represent a species-rich, diverse section. After a thorough comparative analysis of the descriptions it turns out that the only character combination occurring in every species of the section Tricephalium is the character-set of the subgenus Heteropsychotria. The only exception would be the Palicourea neilii C. M. Taylor that has according the description bright yellow flowers and corolla tube swollen and bent at the base, but in his fig. $1 \mathrm{~F}$ the corolla tube is straight and narrow at the base. Therefore we get to the conclusion that the section Tricephalium $\mathrm{C}$. $\mathrm{M}$. Taylor is treated here unchanged as a whole, not in the subgenus Montanae C. M. Taylor, but accentuated its taxonomic position in the frame of the subgenus Heteropsychotria Steyerm.

Key words: Heteropsychotria, new combination, nomenclature, Palicourea, South America, taxonomy

\section{INTRODUCCIÓN}

El subgénero Heteropsychotria fue reconocido y descrito por Julián Steyermark experto sobresaliente de la familia Rubiaceae en la serie monográfica sobre la Botany of the Guyana Highland (1972). Esta elaboración monográfica de la familia Rubiaceae fue publicado por él por la segunda vez en la Flora de Venezuela (1974) en idioma español, con descripciones más detalladas y más ricamente ilustradas. Dentro del subgénero distinguió 194 especies clasificándolas en 13 secciones y 31 series. Tres de las 13 secciones (las de número 4, 7 y 11) no han sido denominadas ni caracterizadas en decripción. Después de haber publicado el estudio molecular fundamental de Nepokroeff el al. (1999), aparecieron una serie de publicaciones monográficas críticas, separando algunas secciones del sistema de Steyermark cómo géneros individuales distintos, que posteriormente resultaron ser válidos. Así, la sección Chytropsia (Bremek.) Steyerm. está incluído en el género Margaritopsis Ch. Wright (Andersson 2001, Taylor 2005). La sección Notopleura recobró su estado genérico, siguiendo el concepto original de Bremekamp (Taylor 2001). La sección Mapouria fue eliminada a consecuencia del re-establecimiento del género Carapichea (Andersson 2002b, Taylor y Gereau 2013). Recientemente, C. M. Taylor reconoció que la mayor parte de las especies clasificadas por Steyermark en su sección Durifolia pertenece al género ampliado Coccochondra (2011).

La reconsideración dela posición taxonómica de subgénero Heteropsychotria adelgazado por los cambios especificados arriba, empezó relativamente tarde en una publicación de Taylor et al. (2010), con la transferencia de algunas especies Mesoamericanas, aunque su relación estrecha ya fue acentuada por 
Nepokroeff et al. (1999). El paso siguiente fue hecho por Borhidi (2011), cuando para la segunda edición de las Rubiáceas de México realizó la transferencia de las especies Mexicanas de Heteropsychotria al género Palicourea, mientras C. M. Taylor mantenía invariadamente las Mesoamericanas dentro del género Psychotria (2012). Recientemente C. M. Taylor empezó revidear la posición taconómica de las especies Sudamericanas del subgénero Heteropsychotria con una nueva circunscripción y re-descripción de la sección Didymocarpae (2014) con 14 especies, con la distinción y creación de una sección nueva, la Tricephalium C. M. Taylor con 35 especies separada del resto de la sección Pseudocephaëlis, y con la elaboración detallada de la sección Nonatelia originalmente monotípica, grandemente extendida y re-considerada en nueva concepción con 26 especies.

\section{LA CIRCUNSCRIPCIÓN ACTUAL DEL SUBGÉNERO HETEROPSYCHOTRIA}

Estamos convencidos que después de tantos cambios enumerados arriba y sufridos por el sistema original de Steyermark sería necesario establecer una circunscripción del subgénero Heteropsychotria dentro del marco del género Palicourea Aubl. proponiendo las consideraciones siguientes. El nuevo sistema propuesto aqui en su fase actual consta de 11 secciones, de las cuales 6 son nuevas.

La primera sección es la Heteropsychotria original notablemente ampliada (42 especies) por la inclusión de la sección anónima no. 4 de Steyermark.

La segunda sección es la Didymocarpae nuevamente descrita por C. M. Taylor, para reemplazar la sección Didymocarpos Steyermark, abarcando aqui 20 especies.

La tercera sección es nueva, descrita cómo Bracteiflorae Borhidi, con 10 especies y Palicourea caerulea, cómo tipo, y comprende la mayor parte de la sección anónima no. 7 de Steyermark después de la salida de las especies separadas al género Carapichea.

La cuarta sección Nonatelia, originalmente monotípica con Palicourea racemosa (Aubl.) Borhidi cómo tipo, ha sido reconsiderada y grandemente extendida por Taylor y Hollowell (2016) en un concepto nuevo con 26 especies.

La quinta sección es la Pseudocephaëlis de Steyermark con 50 especies, casi invariada, solamente la serie Appunianae ha sido separada y transferida a la sección 9. que es la nueva Tricephalium descrita por C. M. Taylor incluyendo 35 especies.

La sexta sección Potaroenses de Steyermark con 6 especies sigue sin cambio alguno. 
La septima y la octava secciones son descendientes de la sección Cephaëlis de Steyermark, que siguen estando separadas en nuestro tratamiento. Las especies con inflorescencias terminales pertenecen a la sección Neocephaëlis Borhidi cómo sección nueva con 17 especies y Palicourea tomentosa (Aubl.) Borhidi, cómo tipo. No es imposible, que mantener el nombre Cephaëlis en sentido reducido, sería incorrecto u erróneo, porque durante 200 años hasta hoy este nombre ha sido utilizado por tantos autores en muchos rangos y sentidos, que hay peligro, que fuera considerado cómo un nomen dubium.

La octava sección comprende especies con inflorescencias axilares sentadas bajo el nombre Axillares, tratada cómo series por Steyermark y elevada aqui al rango de sección con 11 especies y Palicourea axillaris (Aubl.) Borhidi, cómo tipo.

La sección nona es la nueva Tricephalium C. M. Taylor, que vamos a discutir más detalladamente abajo.

La décima sección es la nueva Cordifoliae Borhidi y se formó del resto de la sección Durifolia de Steyermark, con 2 especies y Palicourea cardiomorpha (C. M. Taylor) Borhidi cómo tipo, mientras la sección Oppositiflorae Benth. et Hook. es de ser suprimida, porque su especie típica pertenece a un otro género y es Ronabea latifolia Aubl.

Al final, tenemos una sección nueva conocida por el momento de las Antillas. Esta es la sección Microphyllae Borhidi con 7 especies y Palicourea orientensis Borhidi et Oviedo, cómo tipo. Las especies pertenecientes acá, tienen una combinación de caracteres xeromórfos, hojas pequeñas, inflorescencias sésiles paucifloras.

El subgénero Heteropsychotria de género Palicourea tiene en este tratamiento 11 secciones y más de 220 especies.

\section{LA SITUACIÓN TAXONÓMICA DEL SUBGÉNERO HETEROPSYCHOTRIA}

Taylor intentó eliminar el subgénero Heteropsychotria Steyermark y sumergirlo en sinonimía de subgénero Palicourea. Esta acción no es aceptable, porque los dos subgéneros son diferentes en tener caracteres morfológicos y fisiológicos notablemente diferentes.

El subgénero Palicourea esta caracterizado por tener flores de color brillantes, desodorantes mayormente pediceladas y separadas con corolas largas de tubo a menudo encorvado y abultado en la base con amplio contenido de néctar, protejido por un anillo interno de pelos rígidos, es decir con un complejo arreglado de caracteres adaptado a la polinización de picaflores. La especie típica es Palicourea guianensis Aubl. Por el contrario, el subgénero Heteropsychotria esta caracterizado por tener generalmente inflorescencias verdo- 
sas o blancas con flores blancas, verdosas o flavescentes, fragantes menores y mayormente sésiles, con corolas de tubo derecho, corto, sin abultamiento $\mathrm{y}$ anillo interior de pelos rígidos, pero variadamente pubescente en o sobre la mitad del tubo por dentro. Este complejo arreglado de los caracteres sirve claramente para la polinización por insectos. La especie típica es Palicourea deflexa (Sw.) Borhidi. Taylor se refiere a una gran variación de los caracteres individuales mezcladamente occurridas entre los dos complejos en las especies de la Flora de Guyana Venezolana (2004), pero sin mencionar un ejemplar concreto. Realmente, no hay ningun taxon teniendo un complejo de caracteres intermediario entre los dos subgéneros.

Después de haber sustituido y sinonimizado el subgénero Heteropsychotria Steyerm. Taylor introdujó creando una sección nueva bajo el nombre Tricephalium (Müll. Arg.) C. M. Taylor incluyendo 35 especies y clasificándola en el subgénero Montanae Taylor 1997 de Palicourea. Según la descripción la sección Tricephalium esta diagnostizada por tener hojas de textura engrosada con una reticulación interveniales ricamente evolucionada e inflorescencias con flores sésiles agrupadas en cabezuelas pequeñas o grandes incluídas entre brácteas bien desarrolladas, y con frutos amarillos a rojos en immadurez. Cómo caracteres adicionales están mencionados las estípulas brevemente connadas alrededor del tallo, o laminares, bilobadas a emarginadas, redondeadas, redondeadas y denticuladas, erodadas en un lado, etc.

Realmente, la sección Tricephalium es problemática desde varios puntos de vista. Ella está heterógena por ser una unidad taxonómica, porque no tiene una combinación de caracteres fija, ni un sólo caracter válido para todas las especies del conjunto para distinguirla de las demás secciones. Entre todos sus carecteres morfológicos lo más común puede considerarse la textura y venación de las hojas con la rica reticulación de las venas intersecundarias; pero este es un carácter vegetativo, uno de los más modificables por el cambio de las condiciones ecológicas, es decir, son poco estables. Además, tenemos que tomar en consideración, que la mayoría de las especies tratadas viven en los Andes de Colombia, Ecuador, Peru y Bolivia cómo endémicos locales aislados entre condiciones ambientales montanas muy parecidas. No sabemos, que los caracteres vegetativos cómo van a reaccionar al llegar a un ambiente llano. (Véase al estudio clásico sobre la Achillea lanuginosa por Clausen et al. 1948). Hay que tener en cuenta también, que los caracteres de la textura-venación de las hojas, no son particularidades exclusivas de la especies de Tricephalium, sino se encuentran en otros géneros, p.e. en Carapichea o en Coccochondra también. Otro punto débil de la separación de la sección Tricephalium es la selección superficial del tipo de la sección que es la Palicourea triadica (Müll. Arg.) C. M. Taylor, que es una especie sumamente rara y poco conocida para ser un representante confiable de una sección muy diversa y multispecífica. Hacien- 
do un estudio morfológico comparativo de todas las especies clasificadas en la sección Tricephalium llegamos a la conclusión, que la única combinación de los caracteres, que se encuentran en todas las especies de la sección, es aquel complejo de las propiedades, que caracteriza el subgénero Heteropsychotria. Palicourea neilii C. M. Taylor parecía cómo la única excepción, que tuviera corola amarilla brillante con tubo curvado e inflado en la base, según el protólogo, pero en la fig. 1F la corola de la especie tiene un tubo recto y delgado en la base. Por eso seguimos pensando, que la sección Tricephalium C. M. Taylor - si la mantenemos sin cambiar - no puede ser taratado cómo miembro del subgénero Montanae C. M. Taylor, sino su puesto taxonómico correcto está indudablemente en el marco del subgénero Heteropsychotria Steyerm.

\section{RESEÑA SISTEMÁTICA}

\section{Palicourea Aubl. subgen. Heteropsychotria (Steyerm.) Borhidi, comb. nova}

Basionym: Psychotria L. subgen. Heteropsychotria Steyerm., Mem. N. Y. Bot. Gard. 23: 484 (1972), p. maj. p.

Plantae frutices vel arboreae, rariter herbaceae, stipulae infra apicem ramosum persistentes, virides plerumque bilobatae vel bidentatae, rariter erosae, sine apendice interpeciolar, stipularum cicatricibus sine pilis fimbrillatis ferrugineis; inflorescentiis multiformis plerumque terminalibus, bracteis prominentibus vel minutis; fructi maturi plerumque nigri, glauci, violacei, rariter rubri; pyrenae plerumque ellipticosemiglobosae dorsali convexae et longitodinaliter 3-5 sulcatae vel leves, ventraliter unicarinatae, vel planae et logitudinaliter sulcatae vel esulcatae, rariter fructibus in baccis didymis, globosis, pyrenis dorso sublaevibus vel leviter costatis.

Typus subgeneris: Palicourea deflexa (DC.) Borhidi, Acta Bot. Hung. 53: 243 (2011).

Sectiones exclusae: Chytropsia (Bremekamp) Steyerm., Mem. N. Y. Bot. Gard. 23: 484 (1972) = Margaritopsis Ch. Wright, Anales Acad. Ci. Méd. Habana 6: 146 (1869).

Notopleura Benth., Oerst. Central Amer. Rub. p. 13 (1852), pro sect. $=$ Notopleura (Hook. f.) Bremek., Recueil Trav. Bot. Néerl. 31: 289 (1934), pro gen.

Mapouria (Aubl.) Müll. Arg. in Mart., Fl. Brasil. p.p. = Carapichea Aubl., Hist. Pl. Guiane p. 167 (1775).

Durifolia Steyerm., Mem. N. Y. Bot. Gard. 23: 705 (1972), p. maj. p, = Coccochondra Rauschert, Taxon 31: 561 (1982).

Oppositiflorae Benth. et Hook. Gen. Plant. 2: 124. 1873. = Ronabea Aubl. 
Sección 1. Heteropsychotria Steyerm. emend. Borhidi

Inflorescentiae terminales, paniculatae plerumque multiramosae et multiflorae; flores plerumque sessiles vel subsessiles, solitarii, binatim vel ternatim rariter pluraliter dispositi; pyrenae ventraliter applanatae, dorso semiorbiculariter convexae et longiudinaliter 3-5-sulcatae (42 species).

Typus sectionis: Palicourea deflexa (DC.) Borhidi.

Palicourea adderleyi (Steyerm.) Borhidi, comb. nova, hoc loco - Basionym: Psychotria adderleyi Steyerm., Mem. N. Y. Bot. Gard. 23: 513 (1972). - Holotipo: Brazil, Amazonas Cachoeira Caranguejo, Maguire et al. 60073 (NY). - Ilustr.: Steyermark, Fl. Venez. 3: 1305, fig. 201 (1974).

Palicourea agnata (DC.) Borhidi, Acta Bot. Hung. 57: 274 (2015) - Basionym: Psychotria agnata DC., Prodr. 4: 505 (1830). - Holotipo: Hispaniola, Haiti, colector desconocido (G-DC!).

Palicourea amplectens (Benth.) Delpr. et J. H. Kirkbr., J. Bot. Res. Inst. Texas 10: 412 (2016) - Basionym: Psychotria amplectans Benth. in Hook., Journ. Bot. 3: 230 (1841). - Holotipo: Brazil, Río Branco, Schomburgk 879 (P). - Ilustr.: Steyermark, Fl. Venez. 3: 1324, fig. 206 (1974).

Palicourea araguana (Standl.) Borhidi, comb. nova, hoc loco - Basionym: Psychotria araguana Standl., Field Mus. Publ. Bot. 8: 68 (1930). - Tipo: Venezuela, Aragua, Pittier 11828 (K). - Ilustr.: Steyermark, Fl. Venez. 3: 1292, fig. 197 (1974).

Palicourea aschersoniana (K. Schum. et Kr.) Borhidi, comb. nova, hoc loco - Basionym: Psychotria aschersoniana K. Schum. et Kr., Bot. Jahrb. 40: 330 (1908). - Tipo: Colombia, El Cauca, Cerro Monchique, Lehmann 3722.

Palicourea barnebyana (Steyerm.) Borhidi, comb. nova, hoc loco - Basionym: Psychotria barnebyana Steyerm., Mem. N. Y. Bot. Gard. 23: 542 (1972). Holotipo: Venezuela, Amazonas Piedra Tururumeri, Maguire et al. 37483 (NY).

Palicourea berteroana (DC.) Borhidi, Acta Bot. Hung. 53: 243 (2011) Basionym: Psychotria berteroana DC., Prodr. 4: 515 (1830). - Holotipo: La Española, Bertero s.n. (G-DC!). - Ilustr.: Steyermark, Fl. Venez. 3: 1357, fig. 215 (1974); Burger y Taylor, Fieldiana Bot. n.s. 33: 73, t. 58 (1993); Borhidi, Rub. Mex. 2. ed., p. 377, fig. 84 (2012).

Palicourea bertieroides (Wernh.) Borhidi, comb. nova, hoc loco - Basionym: Psychotria bertieroides Wernham, Bull. Misc. Inform. Kew 1914: 67 (1914). - Tipo: Colombia, Mocoa, Sprague 386 (K).

Palicourea bostrychothyrsus (Sandw.) Delpr. et J. H. Kirkbr., J. Bot. Res. Inst. Texas 10: 414 (2016) - Basionym: Psychotria bostrychothyrsus Sandw., Kew Bull. 1939: 353 (1940). - Holotipo: Guyana Inglesa, Anandabaru, Alston 470 (K). - Ilustr.: Steyermark, Fl. Venez. 3: 1282, fig. 194 (1974). 
Palicourea brachystigma Urb., Ark. Bot. 17(7): 58 (1921) - Holotipo: Haiti, Ma Blanche, Ekman H. 515 (S!). - Syn.: Psychotria deflexa DC. subsp. cubensis Steyerm., Mem. N. Y. Bot. Gard. 23: 505 (1972); Psychotria patens Acuña et Roig in Alain, Flora de Cuba 5: 105 (1962), non Sw. (1788); Psychotria cubensis (Steyerm.) Borhidi, Acta Bot. Hung. 37: 86 (1992).

Palicourea corymbosa (Sw.) Borhidi, Acta Bot. Hung. 57: 274 (2015) - Basionym: Psychotria corymbosa Sw., Prodr. Veg. Ind. Occ. 44 (1788). - Holotipo: Jamaica (S!).

Palicourea costularia (Baill.) Borhidi, comb. nova, hoc loco - Basionym: Uragoga costularia Baill., Adansonia 12: 248 (1879). - Syn.: Psychotria costularia Standl et Steyerm., Fieldiana Bot. 28: 1126 (1957). - Tipo: Venezuela, Aragua, Moritz 851. - Ilustr.: Steyermark, Fl. Venez. 3: 1342, fig. 211 (1974).

Palicourea deflexa (DC.) Borhidi, Acta Bot. Hung. 53: 243 (2011) - Basionym: Psychotria deflexa DC., Prodr. 4: 510 (1830). - Holotipo: Guyana Francesa, Patris s.n. (G-DC!). - Syn.: Psychotria patens auct. non Sw. - Ilustr.: Steyermark, Fl. Venez. 3: 1285, fig. 195 (1974); Burger y Taylor, Fieldiana Bot. n.s. 33: 71, t. 56 (1993); Borhidi, Rub. Mex. 2. ed., p. 382, fig. 85 (2012).

Palicourea domatiata (C. D. Adams) Borhidi, Acta Bot. Hung. 57: 275 (2015) - Basionym: Psychotria domatiata C. D. Adams, Phytologia, 21: 69 (1971). - Holotipo: Jamaica (BM!).

Palicourea domingensis (Jacq.) DC., Prodr. 4: 529 (1830) - Basionym: Psychotria domingensis Jacq., Enum. Pl. Carib. 16 (1760). - Tipo: Santo Domingo, Jacquin. - Syn.: Psychotria pavetta Sw., Prodr. Ind. Veg. p. 45 (1797); Psychotria westii DC., 4: 516 (1830); Porto Rico. Palicourea tabernaefolia DC., Prodr. 4: 525 (1830); Psychotria mombachensis Standl., Publ. Field Mus. Nat. Hist., Bot. Ser. 8: 188 (1930).

Palicourea duricoria (Standl. et Steyerm.) Borhidi, comb. nova, hoc loco - Basionym: Psychotria duricoria Standl. et Steyerm., Fieldiana Bot. 28: 599 (1953). - Holotipo: Venezuela, Amazonas, Cerro Duida, Caño Negro, Steyermark 58048 (NY). - Ilustr.: Steyermark, Fl. Venez. 3: 1353, fig. 214 (1974).

Palicourea erythrocephala (K. Schum. et Kr.) Borhidi, comb. nova, hoc loco - Basionym: Uragoga erythrocephala K. Schum et Kr., Bot. Jahrb. 40: 343 (1908). - Syn.: Psychotria erythrocephala (K. Schum. et Kr.) Standl., Publ. Field Columb. Mus., Bot. Ser. 7: 93 (1930).

Palicourea everardi (Wernh.) Borhidi, comb. nova, hoc loco - Basionym: Psychotria everardi Wernh., Joun. Bot. 52: 216 (1914). - Tipo: Venezuela, Bolivar, Roraima, Im Thurn 291. - Ilustr.: Steyermark, Fl. Venez. 3: 1367, fig. 217 (1974).

Palicourea farameoides (Bremek.) Borhidi, comb. nova hoc loco - Basionym: Psychotria farameoides Bremek., Acta Bot. Neerl. 11: 193 (1962). - Tipo: Surinam, Jodensavanna, Heyligers 573. 
Palicourea glandulicalyx (Steyerm.) Borhidi, comb. nova, hoc loco - Basionym: Psychotria glandulicalyx Steyerm., Mem. N .Y. Bot. Gard. 23: 536, fig. 73 (1972). - Holotipo: Venezuela, Bolivar, Torono-tepui, Steyermark y Wurdack 640 (NY). - Ilustr.: Steyermark, Fl. Venez. 3: 1362, fig. 216 (1974).

Palicourea goyazensis (Müll. Arg.) Borhidi, comb. nova, hoc loco - Basionym: Psychotria goyazensis Müll. Arg., Flora 59: 551 (1876). - Syn.: Psychotria argoviensis Steyerm., Mem. N. Y. Bot. Gard. 23: 500 (1972). - Tipo: Brasil, Pará, Burchell 9227 (NY).

Palicourea guaremalensis (Standl.) Borhidi, comb. nova, hoc loco - Basionym: Psychotria guaremalensis Standl., Field Mus. Publ. Bot. 8: 70 (1930). - Tipo: Venezuela, Upper Guaremales, Pittier 8989. - Ilustr.: Steyermark, Fl. Venez. 3: 1295, fig. 198 (1974).

Palicourea hebeclada (DC.) Borhidi, Acta Bot. Hung. 53: 245 (2011) - Basionym: Psychotria hebeclada DC., Prodr. 4: 513 (1830). - Holotipo: México, Haenke s.n. (PR). - Ilustr.: Burger y Taylor, Fieldiana Bot. n.s. 33: 70, t. 55 (1993). - Syn.: Psychotria molliramis (K. Schum. et K. Krause) Steyerm., Mem. N. Y. Bot. Gard. 23: 529 (1972).

Palicourea imthurniana (Oliver) Borhidi, comb. nova, hoc loco - Basionym: Psychotria imthurniana Oliver, Trans. Linn. Soc. Bot. II. 2: 276, pl. 42, fig. 1-7 (1887). - Tipo: Venezuela, Bolivar, Roraima, Everard 163. - Ilustr.: Steyermark, Fl. Venez. 3: 1324, fig. 206 (1974).

Palicourea liogieri (Steyerm.) Borhidi, Acta Bot. Hung. 57: 275 (2015) Basionym: Psychotria liogieri Steyerm., Mem. N. Y. Bot. Gard. 23: 700 (1972). - Tipo: Haiti, in Fonds Verettes, Buch 1236 (NY). - Syn.: Uragoga domingensis Urb., Repert. Spec. Nov. Regni Veg. $17: 7$ (1921). - Cephaëlis domingensis (Urb.) Standl., Publ. Field Mus. Nat. Hist. Bot. Ser. 4: 335 (1929).

Palicourea longicuspis (Müll. Arg.) Borhidi, Acta Bot. Hung. 59: 18 (2017) - Basionym: Psychotria longicuspis Müll. Arg., Flora 59: 549, 552 (1876). - Isotipo: Brasil, Spruce 2871 (BM). - Syn.: Psychotria cincta Standl., Publ. Field Columb. Mus., Bot. Ser. 7: 90 (1930); Psychotria loretensis Standl., Publ. Field Columb. Mus., Bot. Ser. 8: 198 (1930). - Ilustr.: Steyermark, Fl. Venez. 3: 1297, fig. 199 (1974); Burger y Taylor, Fieldiana Bot. n.s. 33: 73, t. 58 (1993).

Palicourea longirostris (Rusby) Borhidi, comb. nova, hoc loco - Basionym: Rudgea longirostris Rusby, Descr. S. Amer. Pl.: 143 (1920). - Isotipo: Colombia, Smith 1819 (MO). - Syn.: Psychotria longirostris (Rusby) Standl., Publ. Field Columb. Mus., Bot. Ser. 7: 101 (1931). - Ilustr.: Steyermark, Fl. Venez. 3: 1297, fig. 199 (1974).

Palicourea luxurians (Rusby) Borhidi, Acta Bot. Hung. 59: 18 (2017) Basionym: Psychotria luxurians Rusby, Mem. Torrey Bot. Club 6: 50 (1896). Isolectotipo: Bolivia, Bang 1741 (MO). - Ilustr.: Burger y Taylor, Fieldiana Bot. n.s. 33: 72, t. 57 (1993). 
Palicourea manausensis (Steyerm.) Borhidi, comb. nova, hoc loco - Basionym: Psychotria manausensis Steyerm., Mem. N. Y. Bot. Gard. 23: 516 (1972). - Holotipo: Brazil, Amazonas, Manaos, Ule 5386 (HBG).

Palicourea meridensis (Steyerm.) Borhidi, comb. nova, hoc loco - Basionym: Psychotria meridensis Steyerm., Mem. N. Y. Bot. Gard. 23: 531 (1972). Holotipo: Venezuela, Mérida, Tabay, Steyermark 56614 (NY). - Ilustr.: Steyermark, Fl. Venez. 3: 1347, fig. 212 (1974).

Palicourea microbotrys (Ruiz ex Standl.) Borhidi, comb. nova, hoc locoBasionym: Psychotria microbotrys Ruiz ex Standl., Publ. Field Columb. Mus., Bot. Ser. 8: 204 (1930). - Holotipo: Perú, La Victoria, Loreto, Williams 2983 (F). - Ilustr.: Steyermark, Fl. Venez. 3: 1300, fig. 200 (1974); Burger y Taylor, Fieldiana Bot. n.s. 33: 73, t. 58 (1993).

Palicourea nematostachya (Steyerm.) Borhidi, comb. nova, hoc loco - Basionym: Psychotria nematostachya Steyerm., Mem. N. Y. Bot. Gard. 23: 512 (1972). - Holotipo: Brazil, Amazonas, La Neblina, Ewel 114 (NY).

Palicourea obliquinervia (Müll. Arg.) Borhidi, comb. nova, hoc loco - Basionym: Psychotria obliquinervia Müll. Arg., Flora 59: 549, 552 (1876). - Tipo: Brazil, Amazonas, Río Negro, Martius.

Palicourea pakaraimensis (Steyerm.) Borhidi, comb. nova, hoc loco-Basionym: Psychotria pakaraimensis Steyerm., Mem. N. Y. Bot. Gard. 23: 509 (1972). - Holotipo: Guyana Inglesa, Parakaima Mts, Maguire et al. 40573 (NY).

Palicourea pariensis (Steyerm.) Borhidi, comb. nova, hoc loco - Basionym: Psychotria pariensis Steyerm., Mem. N. Y. Bot. Gard. 23: 513 (1972). - Holotipo: Venezuela, Sucre, Peninsula de Paría, Steyermark 95019 (VEN).

Palicourea polymorpha (Greuter) Borhidi et Oviedo, Acta Bot. Hung. 57: 276 (2015) - Basionym: Psychotria polymorpha Greuter, Bissea 3 (num. esp.) 93 (2009). - Holotipo: Cuba, Pico Potrerillo, Alain 6398 (LS/HAC). - Syn.: Psychotria martii Acuña et Roig, Brittonia 14: 224 (1962), nom. illeg.; Psychotria polymorpha Govaerts, Smiths. Contr. Bot. 98: 833 (2012), nom. superfl.

Palicourea pubescens (Sw.) Borhidi, Acta Bot. Hung. 53: 246 (2011) - Basionym: Psychotria pubescens Sw., Prodr. Veg. Ind. Occ. p. 44 (1788). - Holotipo: Jamaica, Swartz s.n. (S!). - Syn.: Psychotria mollis Poir. in Lam., Encycl. Méth. Bot. 5: 701 (1804); Psychotria scabriuscula Bartl. ex DC., Prodr. 4: 513 (1830); Psychotria justicioides Schltdl., Linnaea 9: 596 (1834); Psychotria glauca Polak., Linnaea 41: 569 (1877).

Palicourea pullei (Bremek.) Borhidi, comb. nova, hoc loco - Basionym: Psychotria pullei Bremek., Recueil Trav. Bot. Néerl. 31: 301 (1934). - Tipo: Surinam, Fluv. Saramacca, Pulle 17.

Palicourea ramiflora (Rusby) Borhidi, comb. nova, hoc loco - Basionym: Psychotria ramiflora Rusby, Mem. N. Y. Bot. Gard. 7: 377 (1927). - Syn.: Psychot- 
ria subpedicellata Steyerm., Mem. N. Y. Bot. Gard. 23: 507 (1972). - Holotipo: Perú, Loreto, Klug 2019 (NY).

Palicourea sandwithii Borhidi, nomen novum - Nombre reemplazado: Psychotria kaieteurensis Sandwith, Bull. Misc. Inform. Kew 1939: 552. - Tipo: Guyana Inglesa, Potaro River, Sandwith 1429 (K), non Palicourea kaieteurensis (Wernh.) C. M. Taylor, Novon 24(1): 55-95 (2015).

Palicourea subundulata (Benth.) Borhidi, comb. nova, hoc loco - Basionym: Psychotria subundulata Benth. in Hook., Bot. Journ. 3: 227 (1841). - Tipo: Brasil, Río Negro, Schomburgk 972 (P). - Ilustr.: Steyermark, Fl. Venez. 3: 1290, fig. 196 (1974).

Palicourea surinamensis (Bremek.) Borhidi, comb. nova, hoc loco - Basionym: Psychotria surinamensis Bremek., Recueil Trav. Bot. Néerl. 31: 302 (1934). - Tipo: Surinam, Emma Range, Gonggrijp y Stahel 5735.

Sección 2. Didymocarpeae C. M. Taylor, Novon 23(4) 452 (2014 publ. 2015)

Syn.: Didymocarpos Steyerm., Mem. N. Y. Bot. Gard. 23: 516 (1972), p.p.

Ab aliis sectionibus Heteropsychotriae fructu didymo, subgloboso atque pyrenis subsphaericis, dorso subteretibus convexis laevibus, parietibus tenuibus, atque corolla basi leviter inflata differt. (20 species).

Typus sectionis: Palicourea acuminata (Benth.) Borhidi.

Palicourea acuminata (Benth.) Borhidi, Acta Bot. Hung. 53: 243 (2011) - Basionym: Psychotria acuminata Benth., Bot. Voy. Sulphur p. 107 (1844). Holotipo: Colombia, Isla de Gorgona, Hinds y Sinclair 373 (BM!). - Ilustr.: Burger y Taylor, Fieldiana Bot. n.s. 33: 71, t. 56 (1993).

Palicourea andina C. M. Taylor, Novon 23: 462 (2014 publ. 2015) - Holotipo: Ecuador, Zamora-Chinchipe, Valladolid, Harling y I. Andersson 21405 (MO).

Palicourea andina subsp. panamensis C. M. Taylor, Novon 23: 464 (2014 publ. 2015) - Holotipo: Panama, Chiriquí, vic. Fortuna Dam, McPherson 10427 (MO).

Palicourea boraginoides (Dwyer) C. M. Taylor, Novon 23: 465 (2014 publ. 2015) - Basionym: Psychotria acuminata subsp. boraginoides Dwyer, Ann. Missouri Bot. Gard. 67: 344 (1980). - Holotipo: Panamá, Liesner 1187 (MO).

Palicourea candelabrum (Standl.) C. M. Taylor, Novon 23: 465 (2014 publ. 2015) - Basionym: Psychotria candelabrum Standl., Publ. Field Mus., Bot. Ser. 8: 207 (1930). - Holotipo: Perú, Loreto, Victoria on Amazon, Llewelin Williams 2702 (F). 
Palicourea ceratantha (Standl.) C. M. Taylor, Novon 23: 465 (2014 publ. 2015) - Basionym: Psychoria ceratantha Standl., Field Mus. Publ. Bot. 7: 439. 1931. - Tipo: Venezuela, Amazonas, Mount Duida, Tate 669 (NY). - Syn.: Psychotria arenaria Standl. et Steyerm., Fieldiana Bot. 28: 594 (1953); Psychotria yutajensis Steyerm., Ann. Missouri Bot. Gard. 75: 348 (1988).

Palicourea compta (Standl.) C. M. Taylor, Novon 23: 466 (2014 publ. 2015) - Basionym: Psychotria compta Standl., Publ. Field Columb. Mus., Bot. Ser. 8: 202 (1930). - Holotipo: Perú, Loreto, Yurimaguas, Killip y A. C. Smith 29098 (F).

Palicourea cuspidata (Bredem. ex Schult.) C. M. Taylor, Novon 23: 467 (2014 publ. 2015) - Basionym: Psychotria cuspidata Bredem. in Roem et Schult., Syst. Veg. 5: 192 (1819). - Holotipo: Venezuela, Caracas, Bredemeyer s.n. (BWILLD-4109).

Palicourea cuspidata subsp. occidentalis C. M. Taylor, Novon 23: 467 (2014 publ. 2015) - Holotipo: Colombia, Valle del Cauca, mpio., Yoloco, Reserva Nat. SW de Buga, Devia 3013 (TULV).

Palicourea cuspidulata (K. Krause) C. M. Taylor, Novon 23: 468 (2014 publ. 2015) - Basionym: Cephaëlis cuspidulata K. Krause, Notizbl. Bot. Gart. Berlin-Dahlem 8: 102 (1922). - Holotipo: Perú, Cerro de Escalor, Ule 6551 (B十); lectotipo (F-41047). - Syn.: Psychotria cuspidulata (K. Krause) Standl., Publ. Field Columb. Mus., Bot. Ser. 8: 201 (1930).

Palicourea diminuta C. M. Taylor, Novon 23: 469 (2014 publ. 2015) Holotipo: Guyana, Barima River-head, Eclipse Falls, Pipoly y Lall 8357 (MO).

Palicourea dolichantha (Urb.) Borhidi, Acta Bot. Hung. 57: 275 (2015) Basionym: Psychotria dolichantha Urb., Symb. Ant. 5: 517 (1908). - Holotipo: Jamaica (BM).

Palicourea huampamiensis (C. M. Taylor) C. M. Taylor, Novon 23: 471 (2014 publ. 2015) - Basionym: Psychotria huampamiensis C. M. Taylor, Novon 4: 174 (1994). - Holotipo: Perú, Loreto: Alto Amazonas, Andoas, R. Vásquez y Jaramillo 1896 (MO).

Palicourea jauensis (Steyerm.) C. M. Taylor, Novon 23: 471 (2014 publ. 2015) - Basionym: Psychotria jauensis Steyerm., Mem. N. Y. Bot. Gard. 23: 889 (1972). - Holotipo: Venezuela, Bolivar, Cerro Jáua, Steyermark 98098 (VEN).

Palicourea madidiensis C. M. Taylor, Novon 23: 471 (2014 publ. 2015) Holotipo: Bolivia, La Paz, prov. Nor Yungas, Yolosa, Solomon 8849 (LPB).

Palicourea pandensis (Standl.) C. M. Taylor, Novon 23: 472 (2014 publ. 2015) - Basionym: Psychotria pandensis Standl., Publ. Field Columb. Mus., Bot. Ser. 11: 244 (1936). - Holotipo: Colombia, Prov. Bogotá, Fusagasugá y Pandi, Triana $1709(\mathrm{P})$. 
Palicourea patens (Sw.) Urb., Repert. Spec Nov. Regni Veg. 18: 197 (1922) - Basionym: Psychotria patens Sw., Prodr. Veg. Ind. Occ. p. 45 (1788). - Holotipo: Jamaica (S!).

Palicourea pedunculata (Sw.) Borhidi, Acta Bot. Hung. 57: 276 (2015) - Basionym: Psychotria pedunculata Sw., Prodr. Veg. Ind. Occ. p. 44 (1788). Holotipo: Jamaica (S!).

Palicourea rhodothamna (Standl.) C. M. Taylor, Novon 23: 473 (2014 publ. 2015) - Basionym: Psychotria rhodothamna Standl., Publ. Field Mus., Bot. Ser. 8: 201 (1930). - Holotipo: Perú, Loreto, Iquitos, Killip y A. C. Smith 27115 (F).

Palicourea sanluisensis C. M. Taylor, Novon 23: 474 (2014 publ. 2015) - Holotipo: Colombia, Antioquia, Mpio., San Francisco, Alto del Venado, Fonnegra y Curso Taxonómico, Sem. I/92 4005 (HUA-81193).

Palicourea spicata (Kuntze) C. M. Taylor, Novon 23: 475 (2014 publ. 2015) - Basionym: Uragoga spicata Kuntze, Revis. Gen. Pl. 2: 962 (1891). - Holotipo: Brazil, Amazonas, Rio Negro pr. San Carlos, Spruce 3089 (BR-532-648). - Syn.: Psychotria vaupesana Standl. ex Steyerm., Acta Biol. Venez. 4: 101 (1964).

Palicourea subcuspidata (Müll. Arg.) C. M. Taylor, Novon 23: 475 (2014 publ. 2015) - Basionym: Psychotria subcuspidata Müll. Arg. in C. F. P. von Martius et auct. suc. (eds), Fl. Bras. 6(5): 261 (1881). - Holotipo: Brazil, Amazonas, inter Santarem et Barra do Rio Negro, Spruce Psychotria (3) 991 (BR). - Syn.: Declieuxia psychotrioides DC., Prodr. 4: 481 (1830), non Palicourea psychotrioides (C. M. Taylor et Hammel) C. M. Taylor; Psychotria cornigera Benth., J. Bot. (Hooker) 3: 277 (1841), non Palicourea cornigera C. M. Taylor (1997).

\section{Sección 3. Bracteiflorae Borhidi, sectio nova}

Inflorescentiae elongatae, paniculatae, flores plerumque sessiles vel subsessiles ad ramos laterales in fasciculis terminalibus, bracteis bracteolisque variis plerumque cucullatis, navicularibus vel spathulato-oblanceolatis, cincti. (10 species).

Typus sectionis: Palicourea caerulea (Ruiz et Pav.) Schult. in Roem. et Schult.

Palicourea caerulea (Ruiz et Pav.) Schult. in J. J. Roemer et J. A. Schultes, Syst. Veg., ed. 15 bis 5: 194 (1819) - Basionym: Psychotria caerulea Ruiz et Pav., Fl. Peruv. 2: 62 (1799). - Isotipo: Perú, Ruiz y Pavón s.n. (G-DC!). - Illustr.: Ruiz y Pavón, Fl. Peruv. 2: t. 213 f.b (1799); Steyermark, Fl. Venez. 3: 1450, fig. 238 (1974).

Palicourea egensis (Müll. Arg.) Borhidi, comb. nova, hoc loco - Basionym: Psychotria egensis Müll. Arg., Flora 59: 542, 545 (1876). - Tipo: Brazil, Amazonas Ega, 1831, Poeppig s.n. - Ilustr.: Steyermark, Fl. Venez. 3: 1458, fig. 241 (1974). 
Palicourea hyptoides (Benth.) Borhidi, comb. nova, hoc loco - Basionym: Psychotria hyptoides Benth. in Hook., Journ. Bot. 3: 231 (1841). - Tipo: Guyana Inglesa, Parima Mts, Schomburgk s.n. (K).

Palicourea lourteigiana (Steyerm.) Borhidi, comb. nova, hoc loco - Basionym: Psychotria lourteigiana Steyerm., Mem. N. Y. Bot. Gard. 23: 586, fig. 81, 587 (1942). - Holotipo: Venezuela, Amazonas, Cerro Huachamacari, Maguire et al. 29786 (NY). - Ilustr.: Steyermark, Fl. Venez. 3: 1453, fig. 239 (1974).

Palicourea parvibractea (Steyerm.) Borhidi, comb. nova, hoc loco - Basionym: Psychotria parvibractea Steyerm., Mem. N. Y. Bot. Gard. 23: 581, fig. 80 (1942). - Holotipo: Venezuela, Cerro Venamo, Steyermark y Nilsson 135 (NY). - Ilustr.: Steyermark, Fl. Venez. 3: 1447, fig. 237 (1974).

Palicourea phaneroloma (Standl. et Steyerm.) Borhidi, comb. nova, hoc loco - Basionym: Psychotria phaneroloma Standl. et Steyerm., Fieldiana Bot. 28: 604 (1953). - Tipo: Guyana Inglesa, Cordillera Pakaraima, Schomburgk (P). Ilustr.: Steyermark, Fl. Venez. 3: 1461, fig. 242 (1974).

Palicourea polycephala (Benth.) Borhidi, comb. nova, hoc loco - Basionym: Psychotria polycephala Benth. in Hook., Journ. Bot. 3: 231 (1841). - Tipo: Guyana Inglesa, Schomburgk 139 (P). - Ilustr.: Steyermark, Fl. Venez. 3: 1465, fig. 243 (1974).

Palicourea schomburgkii (Benth.) Borhidi, comb. nova, hoc loco - Basionym: Psychotria schomburgkii Benth. in Hook., Journ. Bot. 3: 231 (1841). - Tipo: Guyana Inglesa, Schomburgk 2085 (P). - Ilustr.: Steyermark, Fl. Venez. 3: 1468, fig. 244 (1974).

Palicourea silvae (Steyerm.) Borhidi, comb. nova, hoc loco - Basionym: Psychotria silvae Steyerm., Mem. N. Y. Bot. Gard. 23: 581 (1972). - Holotipo: Brazil, Amazonas, Serra Pirapucú, Silva y Brazão 60877 (NY). - Ilustr.: Steyerm., Mem. N. Y. Bot. Gard. 23: 582, fig. 79 (1972).

Palicourea wurdackii (Steyerm.) Borhidi, comb. nova, hoc loco-Basionym: Psychotria wurdackii Steyerm., Mem. N. Y. Bot. Gard. 23: 576, fig. 77 (1972). Tipo: Venezuela, Amazonas, Cerro de la Neblina, Maguire y Wurdack 42475 (NY). - Ilustr.: Steyermark, Fl. Venez. 3: 1440, fig. 235 (1974).

\section{Sección 4. Nonatelia (Aubl.) C. M. Taylor, Novon 25: 80. (2016)}

Inflorescentia paniculata elongata et angustata, racemiformis, flores cum lobis corollinis breviter appendiculatis, stylis 5-stigmatibus, bacca 5-partita, pyrenis 5 suffulti (5 species), vel lobis corollinis exappendiculatis atque stylis 2-stigmatibus, bacca 2-partita pyrenis 2 oblati, inflorescentiis spicatis atque paniculatis vel racemiformibus, stipulis varie 2-4-lobatis vel cuspidatis (19 species). 
Species duae: P. locellata C. M. Taylor et P. woronowii (Standl.) C. M. Taylor, combinationis caracterorum a Palicourea sect. Nonatelia diversis verismiliter ad genera adhuc non descripta pertinent.

Typus: Palicourea racemosa (Aubl.) Borhidi

Palicourea amita (Standl.) C. M. Taylor, Novon 25: 83 (2016) - Basionym: Psychotria amita Standl. Field Mus. Publ. Bot. 7: 85 (1931). - Tipo: Colombia, Santander, California, Killip \& Smith 17080 (F). - Ilustr.: Steyermark, Fl. Venez. 3: 1332, Fig. 209 (1974).

Palicourea apiculata (Müll.-Arg.) C. M. Taylor, Novon 25: 84 (2016) - Basionym: Psychotria apiculata Müll.-Arg., Flora 59: 549, 552 (1876). - Tipo: Brazil, Rio de Janeiro, Mandioca, Dec. 1822, L. Riedel 360. p.p.

Palicourea brevicollis (Müll.-Arg.) C. M. Taylor, Novon 25: 85 (2016) Basionym: Psychotria brevicollis Müll.-Arg., Flora 58: 548, 552 (1876). - Tipo: Brazil, Minas Gerais: Caldas, s.d. A.F. Regnell, III, 116. p.p. - Syn.: Psychotria regnellii Müll.-Arg., Flora 58: 548, 552 (1876).

Palicourea cajamarcana C. M. Taylor, Novon 25: 86 (2016) - Holotipo: Peru, Cajamarca: San Ignacio, distr. Huarango, El Triunfo, 1500-1800 m, 13 July 1996, J. Campos et al. 2937 (MO 6404721). - Ilustr.: Novon 25: 77, Fig. 2A-D (2016).

Palicourea carnosocarpa (Dwyer et Hayden) C. M. Taylor, Novon 25: 88 (2016) - Basionym : Psychotria carnosocarpa Dwyer et M. V. Hayden, Ann. Missouri Bot. Gard. 55: 41 (1968). - Holotipo: Panamá, Lewis et al. 964 (MO).

Palicourea dodsoniana C. M. Taylor, Novon 25: 88 (2016) - Holotipo: Ecuador, Los Rios, rd. Santo Domingo-Quevedo km 47, Río Palenque Science Center, $00^{\circ} 35^{\prime} \mathrm{S}, 79^{\circ} 22^{\prime} \mathrm{W}, 200 \mathrm{~m}, 18$. Nov. 1991, C. Persson et al. 63 (MO 04642154). - Ilustr.: Novon 25: 77, Fig. 2E-F (2016).

Palicourea eggersii (Standl.) C. M. Taylor, Novon 25: 89 (2016) - Basionym: Psychotria eggersii Standl., Field Mus. Publ. Bot. 8: 69 (1930). - Tipo: Venezuela, Caracas, Eggers 13187. - Ilustr.: Steyermark, Fl. Venez. 3: 1330, Fig. 208 (1974), Novon 25: 75, Fig. 1I-J (2016).

Palicourea fissistipula (Müll.-Arg.) C. M. Taylor, Novon 25: 90 (2016) - Basionym: Psychotria fissistipula Müll.-Arg., Flora 59: 547, 551 (1876). - Holotipo: Brazil, sin. loc., s.d., F. Sellow, s.n. (B十) (lectotipo: G 00300759).

Palicourea forsteronioides (Müll.-Arg.) C. M. Taylor, Novon 25: 91 (2016) - Basionym: Psychotria forsteronioides Müll.-Arg., Flora 59: 550, 553 (1876). - Tipo: Brazil, Estado Río de Janeiro, H. W. Schott s.n., sintipo: São Paulo, Juqueri, P. W. Lund, s.n.

Palicourea locellata C. M. Taylor Novon 25: 92 (2016) - Holotipo: Colombia, Cundinamarca, mpio. Ubala vereda Campo Hermoso hacia El Retiro, 


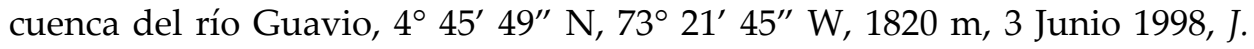
Fernández et al. 16420 (COL-480439). - Ilustr.: Novon 25: 78, Fig. 3A-E (2016).

Palicourea malaneoides (Müll.-Arg.) C. M. Taylor, Novon 25: 93 (2016) - Basionym: Psychotria malaneoides Müll.-Arg., Flora 59. 550, 553 (1876). - Lectotipo: Brazil, sin loc., s.d., J. B. E. Pohl 4365 (G 00300553).

Palicourea mamillaris (Müll.-Arg.) C. M. Taylor, Novon 25: 94 (2016) - Basionym: Psychotria mamillaris Müll.-Arg., Fl. Bras. 6(5): 299 (1881). - Holotipo: Brazil, Río de Janeiro, Itaboraí entre Villa Nova y Porto de Caixas, 11. Feb. 1875, A Glaziou 7687 (G 00300924).

Palicourea meieri C. M. Taylor, Novon 25: 95 (2016) - Holotipo: Venezuela, Miranda, S del caserio El Palacio de la Providencia $19 \mathrm{~km}$ al SW Ocumare del Tuy, $10^{\circ} 02^{\prime} \mathrm{N}, 66^{\circ} 56^{\prime} \mathrm{W}, 100 \mathrm{~m}, 12$ Mayo 1999, A. Narvaez et al. 300 (VEN-433031). - Ilustr.: Novon 25: 75, Fig. 1E-H (2016).

Palicourea minutiflora (Müll.-Arg.) C. M. Taylor, Novon 25: 96 (2016) - Basionym: Psychotria minutiflora Müll.-Arg., Flora 59: 549, 552 (1876). - Lectotipo: Brazil, Bahia; sin. loc., s.d., J. S. Blanchet 3982 (G 00300574).

Palicourea obconica (Müll.-Arg.) C. M. Taylor, Novon 25: 97 (2016) - Basionym: Psychotria obconica Müll.-Arg., Flora 59: 550, 553 (1876). - Lectotipo: Brazil, Amazonas, pr. San Gabriel de Cochoeira ad Río Negro, enero-febrero 1852, R. Spruce 2036 (MO 189149).

Palicourea octocuspis (Müll.-Arg.) C. M. Taylor, Novon 25: 98 (2016) - Basionym: Psychotria octocuspis Müll.-Arg., Flora 59: 548, 551 (1876). - Lectotipo: Brazil, Bahia: Ilheos, s.d., M.A.P. Wied-Neuwied, s.n. (BR 00000659001).

Palicourea racemosa (Aubl.) Borhidi, Acta Bot. Hung. 53: 247 (2011) Basionym: Nonatelia racemosa Aubl., Hist. Pl. Guian., p. 187, tab. 72 (1775). Holotipo: Guyana Francesa, Aublet s.n. (P!). - Syn.: Psychotria racemosa (Aubl.) Rich., Actes Soc. Hist. Nat. Paris 1: 107 (1792). - Ilustr.: Burger y Taylor, Fieldiana Bot. n.s. 33: 74, t. 59 (1993); Borhidi, Rub. Mex. 2. ed., p. 397, fig. 88 (2012).

Palicourea soejartoi (C. M. Taylor) C. M. Taylor, Novon 25: 100 (2016) Basionym: Psychotria soejartoi C. M. Taylor, Novon 14: 506 (2004). - Holotipo: Colombia, Antioquia, mpio. Anorí, Valle Anorí, entre Dos Bocas y Anorí, Buenos Aires, 400-900 m, 6-12 Sep. 1973, D. D. Soejarto et al. 4318 (MO-4030419). - Ilustr.: Taylor, Novon 14: 500, t. 3A (2004).

Palicourea stenostachya (Standl.) C. M. Taylor, Novon 25: 101 (2016) Basionym: Psychotria stenostachya Standl., Field Mus. Nat. Hist. Bot. Ser. 8(3): 207 (1930). - Holotipo: Peru, Loreto, San Antonio on Río Itaya, 110 m, 18. Sep. 1929, E. P. Killip y A. C. Smith 29294 (F-607496). - Ilustr.: Novon 25: 77, Fig. 2G-H (2016).

Palicourea subfusca (Müll.-Arg.) C. M. Taylor, Novon 25: 101 (2016) Basionym: Psychotria subfusca Müll.-Arg., Fl. Bras. 6(5): 297 (1881). - Holotipo: Brazil, Amazonas, Alto Amazonas, Río Negro, s.d., G. Martius s.n. (M-189083). 
Palicourea tacarcunensis (Dwyer) C. M. Taylor, Novon 25: 102 (2016) Basionym: Psychotria tacarcunensis Dwyer, Ann. Missouri Bot. Gard. 67(2): 431 (1980). - Holotipo: Panamá: Darién/Chocó, top of W peak Tacaracuna Massif, 1800-1850 m, 28. enero 1975, A. Gentry y S. Mori 13974 (MO-2242943).

Palicourea topoensis C. M. Taylor, Novon 25: 103 (2016) - Holotipo: Ecuador, Napo, Parque Nac. Sumaco-Galeras, S slope Sumaco volcano, $00^{\circ}$ $37^{\prime} \mathrm{S}, 77^{\circ} 35^{\prime} \mathrm{W}, 1600 \mathrm{~m}, 15$. Oct. 2005, J. Homeier y M. A. Chinchero 1879 (MO6136935). - Ilustr.: Novon 25: 75, Fig. 1A-D (2016).

Palicourea tsakiana (C. M. Taylor) C. M. Taylor, Novon 25: 104 (2016) Basionym: Psychotria tsakiana C. M. Taylor, Novon 21: 146 (2011). - Holotipo: Costa Rica, Limón, Talamanca, bosque de Tsãki, Apr. 1895, A. Tonduz 9579 (US-943477).

Palicourea valerioana (Standl.) C. M. Taylor, Novon 25: 105 (2016) - Basionym: Psychotria valerioana Standl., Publ. Field Columb. Mus., Bot. Ser. 18: 1363 (1938). - Holotipo: Costa Rica, Alajuela, Cerros de San Pedro y de San Ramón, 1075 m, Junio 1926, A. M. Brenes 4886 (F-853470). - Ilustr.: Burger y Taylor, Fieldiana Bot., n.s. 33: 71, t. 56 (1993).

Palicourea veracruzensis (Lorence et Dwyer) Borhidi, Acta Bot. Hung. 53(3-4): 248 (2011) - Basionym: Psychotria veracruzensis Lorence et Dwyer, Bol. Soc. Bot. Mex. 47: 621, fig. 4 (1987). - Holotipo: México, Veracruz, mpio. San Andrés Tuxtla, Estación Biol. Tropica „Los Tuxtlas” entre Sontecomapan y Montepío, vereda al Cerro Vígia, 150-250 m, 18 Apr. 1981, D. Lorence y T. P. Ramamoorthy 3313 (MEXU!).

Palicourea woronowii (Standl.) C. M. Taylor, Bruniera et Zappi, Kew Bull. 70(45): 2, Fig. 2 (2015) - Basionym: Rudgea woronowii Standl., Publ. Field Mus. Nat. Hist. Bot. Ser. 7(1): 155 (1930). - Holotipo: Colombia, Caquetá, ca. Ketuchá, Río Ortegraza, 20 Aug. 1926, G. Woronow 6064 (LE), (isotipo: F-599983). - Ilustr.: Taylor et al., fig. 617 (2004).

\section{Sección 5. Pseudocephaëlis (Steyerm.) Borhidi, comb. nova}

Basionym: Psychotria L. sect. Pseudocephaëlis Steyerm., Mem. N. Y. Bot. Gard. 23: 593 (1972).

Inflorescentiae capitatae vel subcapitatae, monocephalae, vel in umbellis, racemis, corymbis vel cymis dispositae, bracteis extimis involucralibus plerumque conspicuis, evolutis atque liberis, flores individuales non bibracteolati, bracteolae atque bracteae pro floribus solitariae vel nullae. (50 species).

Typus: Palicourea hoffmannseggiana (Willd. ex Roem et Schult.) Borhidi

Palicourea adenophora (Steyerm.) Borhidi, comb. nova, hoc loco - Basionym: Psychotria adenophora Steyerm., Mem. N. Y. Bot. Gard. 23: 599 (1972). 
- Holotipo: Venezuela, Amazonas, Cerro Huachamacari, Maguire et al. 29851 (NY).

Palicourea adpressipilis (Steyerm.) Borhidi, comb. nova, hoc loco - Basionym: Psychotria adpressipilis Steyerm., Mem. N. Y. Bot. Gard. 23: 648 (1972). - Holotipo: Perú, Tarapoto, Spruce 4008 (NY).

Palicourea alba (Aubl.) Delpr. et J. H. Kirkbr., J. Bot. Res. Inst. Texas 10: 412 (2016) - Basionym: Tapogomea alba Aubl., Pl. Guian. 1: 164, pl. 62, fig. 4 (1775). - Tipo: Guyana Francesa, Aublet (P). - Syn.: Geophila picta Rolfe, Kew Bull. 1896: 18 (1896); Psychotria ulviformis Steyerm., Mem. N. Y. Bot. Gard. 23: 638 (1972). - Ilustr.: Steyermark, Fl. Venez. 3: 1552, fig. 267 (1974).

Palicorea ayangannensis (Steyerm.) Delpr. et J. H. Kirkbr., J. Bot. Res. Inst. Texas 10: 413 (2016) - Basionym: Psychotria ayangannensis Steyerm., Mem. N. Y. Bot. Gard. 23: 594 (1972). - Holotipo: Guyana Inglesa, Campo Thomson, Mt Ayanganna, Tillett y Boyan 45059 (NY). - Ilustr.: Steyerm., Mem. N. Y. Bot. Gard. 23: 595, fig. 82 (1972).

Palicourea blakei (Standl. et Steyerm.) Borhidi, comb. nova, hoc loco - Basionym: Psychotria blakei Standl. et Steyerm., Fieldiana Bot. 28: 596, fig. 133 (1953). - Tipo: Venezuela, Amazonas, Cerro Yapacana, Rio Orinoco, Holt y Blake 743. - Ilustr.: Steyermark, Fl. Venez. 3: 1537, fig. 263 (1974).

Palicourea bolivarensis (Standl. et Steyerm) Borhidi, comb. nova, hoc loco - Basionym: Cephaëlis bolivarensis Standl. et Steyerm., Fieldiana Bot. 28: 567 (1953). - Tipo: Venezuela, Bolivar, Sororopán-tepui, Steyermark 60185. - Syn.: Psychotria bolivarensis (Standl. et Steyerm.) Steyerm., Mem. N. Y. Bot. Gard. 23: 651 (1972).

Palicourea brazaoi (Steyerm.) Borhidi, comb. nova, hoc loco - Basionym: Psychotria brazaoi Steyerm., Mem. N. Y. Bot. Gard. 23: 610, fig. 83 (1972). Holotipo: Brazil, Amazonas, Igarapé Anta, Pico da Neblina, Silva y Brazão 60633 (NY). - Ilustr.: Steyermark, Fl. Venez. 3: 1504, fig. 254 (1974).

Palicourea callithrix (Miq.) Delpr. et J. H. Kirkbr., J. Bot. Res. Inst. Texas 10: 414 (2016) - Basionym: Cephaëlis callithrix Miq., Symb. Surin., Linnaea 18: 746 (1844). - Tipo: Surinam, Para, Kappler 1473. - Syn.: Psychotria callithrix (Miq.) Steyerm., Mem. N. Y. Bot. Gard. 23: 640 (1972); Psychotria oiapoquensis Steyerm., Mem. N. Y. Bot. Gard. 23: 647 (1972).

Palicourea campyloneura (Müll. Arg.) Delpr. et J. H. Kirkbr., J. Bot. Res. Inst. Texas 10: 415 (2016) - Psychotria campyloneura Müll. Arg. Mart. Flor. Bras. 6(5): 302. 1881.

Palicourea canescens (Steyerm.) Borhidi, comb. nova, hoc loco - Basionym: Psychotria canescens Steyerm., Mem. N. Y. Bot. Gard. 23: 651 (1972). - Holotipo: Brazil, Serra Pirapucú, Rio Negro, Rio Cauaburi, Silva y Brazão 60880 (NY). Ilustr.: Steyerm., Mem. N. Y. Bot. Gard. 23: 652, fig. 86 (1972). 
Palicourea capitata (Ruiz et Pav.) Borhidi, Acta Bot. Hung. 53: 243 (2011) - Basionym: Psychotria capitata Ruiz et Pav., Fl. Peruv. 2: 59 (1799). - Holotipo: Perú, Ruiz y Pavón s.n. (MA). - Ilustr.: Ruiz y Pavón, Fl. Peruv. 2: t. 206 f.a (1799); Steyermark, Fl. Venez. 3: 1522, fig. 258. (1974).

Palicourea caquetensis (Steyerm.) Borhidi, comb. nova, hoc loco - Basionym: Psychotria caquetensis Steyerm., Mem. N. Y. Bot. Gard. 23: 653 (1972). Holotipo: Colombia, Caquetá, Vogel $81(\mathrm{M})$.

Palicourea casiquiaria (Müll. Arg.) Borhidi, comb. nova, hoc loco - Basionym: Psychotria casiquiaria Müll. Arg. in Mart., Fl. Bras. 6(5): 324 (1881). - Tipo: Venezuela, Amazonas, Casiquiare, Spruce 3436. - Ilustr.: Steyermark, Fl. Venez. 3: 1490, fig. 250 (1974).

Palicourea cerronis (Steyerm.) Borhidi, comb. nova, hoc loco - Basionym: Psychotria cerronis Steyerm., Mem. N. Y. Bot. Gard. 23: 886 (1972). - Tipo: Venezuela, Amazonas, Cerro Jáua, Steyermark 97852. - Ilustr.: Steyermark, Fl. Venez. 3: 1481, fig. 248 (1974).

Palicourea concinna (Oliv.) Borhidi, comb. nova, hoc loco - Basionym: Psychotria concinna Oliver, Trans. Linn. Soc. Bot. II, 2: 276, pl. 42, fig. 8-15 (1887). - Tipo: Venezuela, Bolivar, Roraima, Summit, Connell y Quelch 667. - Ilustr.: Steyermark, Fl. Venez. 3: 1483, fig. 249 (1974).

Palicourea contracta (Müll. Arg.) Borhidi, comb. nova, hoc loco - Basionym: Psychotria contracta Müll. Arg. in C. F. P. von Martius et auct. suc. (eds), Fl. Bras. 6(5): 330 (1881). - Tipo: Brazil, Amazonas, Manaus, Spruce 1814.

Palicourea duidana (Standl.) Borhidi, comb. nova, hoc loco - Basionym: Psychotria duidana Standl., Field Mus. Publ. Bot. 7: 443 (1931). - Tipo: Venezuela, Amazonas, Mt Duida, Tate 713 (F).

Palicourea embirensis (Steyerm.) Borhidi, comb. nova, hoc loco - Basionym: Psychotria embirensis Steyerm., Mem. N. Y. Bot. Gard. 23: 656 (1972). Holotipo: Brazil, Amazonas, basin of Rio Yurua, embocadura de Rio Embira, Krukoff 4940 (NY).

Palicourea fosbergii (Steyerm.) Borhidi, comb. nova, hoc loco - Basionym.: Psychotria fosbergii Steyerm., Mem. N. Y. Bot. Gard. 23: 630 (1972). - Holotipo: Colombia, Santander, Cordillera Oriental, Cuchillo de los Micos, Fosberg y Fassett 21810 (US).

Palicourea glabra (Aubl.) Borhidi, comb. nova, hoc loco - Basionym: Tapogomea glabra Aubl., Pl. Guian. 1: 165, pl. 63 (1775), non Psychotria glabra (Turrill) Fosberg (1942). - Holotipo: Guyana Francesa, Aublet. (P). - Syn.: Psychotria blepharophylla (Stand1.) Steyerm., Mem. N. Y. Bot. Gard. 23: 649 (1972).

Palicourea gracilenta (Müll. Arg.) Borhidi, Acta Bot. Hung. 59: 17 (2017) - Basionym: Psychotria gracilenta Müll. Arg., Flora 59: 545 (1876). - Isotipo: Blanchet 1590 (BM). - Syn.: Psychotria brachybotrya Müll. Arg. in C. F. P. von Martius et auct. suc. (eds), Fl. Bras. 6(5): 327 (1881). - Ilustr.: Steyermark, Fl. 
Venez. 3: 1502, fig. 253 (1974); Burger y Taylor, Fieldiana Bot. n.s. 33: 71, t. 56 (1993).

Palicourea hemicephaëlis (Wernh.) Borhidi, comb. nova, hoc loco - Basionym: Psychotria hemicephaëlis Wernh., Jour. Bot. 52: 314 (1914). - Tipo: Guyana Inglesa, Potaro River, Kaiteur Savanna, Jenman 1223. - Ilustr.: Steyermark, Fl. Venez. 3: 1471, fig. 245 (1974). - Syn.: Cephaëlis hemicephaëlis (Wernh.) Steyerm., Mem. N. Y. Bot. Gard. 17(1): 426, fig. 441a-e (1967).

Palicourea hirta (Miq.) Borhidi, comb. nova, hoc loco - Basionym: Cephaëlis hirta Miq., Linnaea 17: 71 (1843), non Psychotria hirta Humb. et Bonpl. in Roem. et Schult. (1819). - Tipo: Surinam, Bergendaal, Focke 382. - Syn.: Psychotria bremekampiana Steyerm., Mem. N. Y. Bot. Gard. 23: 643 (1972).

Palicourea hoffmannseggiana (Willd. ex Roem. et Schult.) Borhidi, Acta Bot. Hung. 53: 245 (2011) - Basionym: Cephaëlis hoffmannseggiana Willd. ex Roem. et Schult., Syst. Veg. 5: 214 (1819). - Holotipo: Brasil: Pará, Hoffmannsegg s.n. (B-WILLD). - Ilustr.: Steyermark, Fl. Venez 3: 1946, fig. 252 (1974); Burger y Taylor, Fieldiana Bot. n.s. 33: 71, t. 56 (1993).

Palicourea humboldtiana (Cham.) Borhidi, comb. nova, hoc loco - Basionym: Cephaëlis humboldtiana Cham., Linnaea 4: 136 (1829). - Tipo: Venezuela, Amazonas, Javita, Humboldt y Bonpland (P). - Syn.: Psychotria humboldtiana (Cham.) Müll. Arg. in Mart., Fl. Bras. 6(5): 333 (1881). - Ilustr.: Steyermark, Fl. Venez. 3: 1511, fig. 256 (1974).

Palicourea iodotricha (Müll. Arg.) Borhidi, comb. nova, hoc loco - Basionym: Psychotria iodotricha Müll. Arg. in Mart., Fl. Bras. 6(5): 375 (1881). - Tipo: Venezuela, Amazonas, Casiquiari, Spruce 3274. - Ilustr.: Steyermark, Fl. Venez. 3: 1559, fig. 269 (1974).

Palicourea leiantha (Steyerm.) Borhidi, comb. nova, hoc loco - Basionym: Psychotria leiantha Steyerm., Mem. N. Y. Bot. Gard. 23: 635, fig. 85 (1972). Holotipo: Venezuela, Amazonas, Cañon Grande, Cerro de la Neblina, Rio Yatua, Maguire et al. 42449 (NY). - Ilustr.: Steyermark, Fl. Venez. 3: 1549, fig. 266 (1974).

Palicourea lupulina (Benth.) Borhidi, comb. nova, hoc loco - Basionym: Psychotria lupulina Benth., in Hook. Jour. Bot. 3: 230 (1841). - Tipo: Guyana Inglesa, Schomburgk 26 (P). - Ilustr.: Steyermark, Fl. Venez. 3: 1540, fig. 264 (1974).

Palicourea maguireorum (Steyerm.) Borhidi, comb. nova, hoc loco - Basionym: Psychotria maguireorum Steyerm., Mem. N. Y. Bot. Gard. 23: 625, fig. 84. 626 (1972). - Tipo: Venezuela, Amazonas, Casiquiari, Vasiva y Pacimoni, Spruce 3440. - Ilustr.: Steyermark, Fl. Venez. 3: 1559, fig. 260 (1974).

Palicourea manaraeana (Steyerm.) Borhidi, comb. nova, hoc loco - Basionym: Psychotria manaraeana Steyerm., Acta Bot. Venez. 6: 143, fig. 7 (1971); Mem. N. Y. Bot. Gard. 23: 627 (1972). - Holotipo: Venezuela, Lara, Palavecino, 
Loma redondo, Steyermark et al. 103454 (VEN). - Ilustr.: Steyermark, Fl. Venez. 3: 1532, fig. 261 (1974).

Palicourea medusula (Müll. Arg.) Borhidi, comb. nova, hoc loco - Basionym: Psychotria medusula Müll. Arg., Flora 59: 540, 548 (1876); in Mart. Fl. Bras. 6(5): 342 (1881). - Tipo: Brazil, Amazonas, San Gabriel do Cachoeira, Spruce 2597. - Ilustr.: Steyermark, Fl. Venez. 3: 1563, fig. 270 (1974).

Palicourea megacephala (Steyerm.) Borhidi, comb. nova, hoc loco - Basionym: Psychotria megacephala Steyerm., Mem. N. Y. Bot. Gard. 23: 646 (1972). - Holotipo: Brazil, Amazonas, Livramento, Krukoj 6858 (NY).

Palicourea oblita (Wernh.) Borhidi, comb. nova, hoc loco - Basionym: Psychotria oblita Wernh., Jour. Bot. 52: 314 (1914). - Lectotipo: Venezuela, Bolivar, Roraima, Appun 1103. - Ilustr.: Steyermark, Fl. Venez. 3: 1473, fig. 246 (1974). - Syn.: Palicourea roraimae Standl., Field Mus. Publ. Bot. 17: 280 (1937).

Palicourea ostreophora (Wernh.) Borhidi, comb. nova, hoc loco - Basionym: Cephaëlis ostreophora Wernham, J. Bot. 55: 284 (1917). - Tipo: Venezuela, Sucre, Cocollar, Humboldt y Bonpland (P). - Syn.: Evea lucentifolia Blake, Contr. U. S. Nat. Herb. 20: 532, pl. 42 (1924); Psychotria lucentifolia (Blake) Steyerm., Mem. N. Y. Bot. Gard. 23: 610 (1972); Psychotria ostreophora (Wernham) C. M. Taylor, Novon 4: 305 (1994). - Ilustr.: Steyermark, Fl. Venez. 3: 1507, fig. 255 (1974).

Palicourea piresii (Steyerm.) Borhidi, comb. nova, hoc loco - Basionym: Psychotria piresii Steyerm., Mem. N. Y. Bot. Gard. 23: 624 (1972). - Holotipo: Brazil, Amapá, Murça Pires y Cavalcante 52250 (NY).

Palicourea platypoda (DC.) Borhidi, Acta Bot. Hung. 59: 19 (2017) - Basionym: Psychotria platypoda DC., Prodr. 4: 510 (1830). - Holotipo: Guyana Francesa, Patris s.n. (G-DC!).

Palicourea prancei (Steyerm.) Borhidi, comb. nova, hoc loco - Basionym: Psychotria prancei Steyerm., Mem. N. Y. Bot. Gard. 23: 656 (1972). - Holotipo: Brazil, Amazonas, Reserva Forestal Ducke, Prance et al. 9015 (NY).

Palicourea prunifolia (H. B. K.) Borhidi, comb. nova, hoc loco - Basionym: Cephaëlis prunifolia H. B. K., Nov. Gen. et Spec. 3: 377 (1819). - Syn.: Cephaёlis microcephala Willd. ex Roem. et Schult., Syst. Veg. 5: 214 (1819); Psychotria prunifolia (H. B. K.) Steyerm., Mem. N. Y. Bot. Gard. 23: 655 (1972). - Tipo: Venezuela, Amazonas, Orinoco, Maypures, Humboldt 757 (P). - Ilustr.: Steyermark, Fl. Venez. 3: 1569, fig. 272 (1974).

Palicourea pseudinundata (Wernh.) Borhidi, comb. nova, hoc loco - Basionym: Psychotria pseudinundata Wernham, J. Bot. 52: 315 (1914). - Tipo: Guyana Francesa, Patris s.n. - Syn.: Psychotria bracteata DC., Prodr. 4: 510 (1830), not F. G. Dietr. Vollst. Lexic. Gaertn. 7: 617 (1809). 
Palicourea quadribracteata (Steyerm.) Borhidi, comb. nova, hoc loco - Basionym: Psychotria quadribracteata Steyerm., Mem. N. Y. Bot. Gard. 23: 614 (1972). - Holotipo: Brazil, Pará, Belém, Silva 81 (NY).

Palicourea raveniana Borhidi, nomen novum, hoc loco - Nombre reemplazado: Psychotria lindenii Standl., Field Mus. Publ. Bot. 8: 71 (1930), non Palicourea lindenii Standl., Publ. Field Columb. Mus., Bot. Ser. 7: 138 (1930). - Tipo: Venezuela, Mérida, Linden 1419 (F). - Ilustr.: Steyermark, Fl. Venez. 3: 1546, fig. 265 (1974). Dedicado al honor de Peter H. Raven, director anterior de Missouri Botanical Garden.

Palicourea sphaerocephala (Müll. Arg.) Borhidi, comb. nova, hoc loco - Basionym: Psychotria sphaerocephala Müll. Arg., Flora 59: 550, 553 (1876); in Mart., Fl. Bras. 6(5): 340 (1881). - Tipo: Brazil, Manaos, Barra, Spruce 1287. - Syn.: Cephaëlis sphaerocephala B. L. Rob., Proc. Amer. Acad. 45: 408 (1910). - Ilustr.: Steyermark, Fl. Venez. 3: 1566, fig. 271 (1974).

Palicourea stipulosa (Müll. Arg.) Borhidi, comb. nova, hoc loco - Basionym: Psychotria stipulosa Müll. Arg. in Mart., Fl. Bras. 6(5): 334 (1881). - Tipo: Venezuela, Casiquiare, Vasiva y Pacimoni, Spruce 3382. - Syn.: Cephaëlis stipulosa (Müll. Arg.) Standl. - Ilustr.: Steyermark, Fl. Venez. 3: 1526, fig. 259 (1974).

Palicourea tapirapecoana (Steyerm.) Borhidi, comb. nova, hoc loco - Basionym: Psychotria tapirapecoana Steyerm., Mem. N. Y. Bot. Gard. 23: 628 (1972). - Holotipo: Venezuela, Brazil frontera, Amazonas, Rio Castanho afluente del Padauiri, Puig 1496 (VEN). - Ilustr.: Steyermark, Fl. Venez. 3: 1535, fig. 262 (1974).

Palicourea tatei (Standl. in Gleason) Borhidi, comb. nova, hoc loco - Basionym: Cephaëlis tatei Standl. in Gleason, Bull. Torrey Bot. Club 56: 406 (1929), non Psychotria tatei Standl. Field Mus. Publ. Bot. 7: 460 (1931). - Tipo: Guyana Inglesa, Kaieteur Savanna, Sandwith 1390. - Syn.: Psychotria crocochlamys Sandw., Kew Bull. 1939: 555 (1939). - Ilustr.: Steyermark, Fl. Venez. 3: 1476, fig. 247 (1974).

Palicourea tetramera (Steyerm.) Borhidi, comb. nova, hoc loco - Basionym: Psychotria tetramera Steyerm., Mem. N. Y. Bot. Gard. 23: 654 (1972). - Holotipo: Guyana Inglesa, Chinoweg, Forest DEPT. Record no. 7832 (NY).

Palicourea trichocephala (Poepp. in Poepp. et Endl.) Borhidi, comb. nova, hoc loco - Basionym: Psychotria trichocephala Poepp. in E. F. Poeppig et S. L. Endlicher, Nov. Gen. Sp. Pl. 3: 32 (1841). - Tipo: Perú, San Martín, Tocache, Poeppig 2039. - Syn.: Cephaëlis trichocephala (Poepp. in Poepp. et Endl.) Standl., Field Mus. Publ. Bot. 8: 375 (1931).

Palicourea trichophora (Müll. Arg.) Borhidi, comb. nova, hoc loco - Basionym: Psychotria trichophora Müll. Arg. in C. F. P. von Martius et auct. suc. (eds), Fl. Bras. 6(5): 343 (1881). - Tipo: Brazil, Bahia 1831, Blanchet 732. - Syn.: Psychotria sciaphila S. Moore, Trans. Linn. Soc. London, Bot. 4: 379 (1895). 
Palicourea variegata (Steyerm.) Borhidi, comb. nova, hoc loco - Basionym: Psychotria variegata Steyerm., Mem. N. Y. Bot. Gard. 23: 638 (1972). - Tipo: Guyana Francesa, Aublet (P). - Syn.: Tapogomea purpurea Aubl., Pl. Guian. 1: 162, pl. 62, fig. 3 (1775), non Palicourea purpurea C. M. Taylor, Syst. Bot. Monogr. 26: 71 (1989). - Ilustr.: Steyermark, Fl. Venez. 3: 1556, fig. 268 (1974).

Palicourea vichadensis (Standl.) Borhidi, comb. nova, hoc loco - Basionym: Psychotria vichadensis Standl., Field Mus. Publ. Bot. 22: 207 (1940). - Tipo: Colombia, El Vichada, San José de Ocune, Haught 2807. (F). - Ilustr.: Steyermark, Fl. Venez. 3: 1514, fig. 257 (1974).

Sección 6. Potaroenses (Steyerm.) Borhidi, comb. nova sub Palicourea Aubl.

Basionym: sect. Potaroenses sub Psychotria L., Mem. N. Y. Bot. Gard. 23: 662 (1972).

Flores in capitulis compactiformibus dispositi, ebracteolatique ebracteati, corollae tubus intus basin versus annulo pilorum instructus; flores longistyli stigmatibus suborbicularibus instructi, antheris apiculatis, stipula subfloral suprema inter dentes laterales apendicem elongatam centralem gerens. (6 species).

Typus: Palicourea potaroensis (Sandw.) Borhidi.

Palicourea aetantha (Sandw.) Delpr. et J. H. Kirkbr., J. Bot. Res. Inst. Texas 10: 412 (2016) - Basionym: Cephaëlis aetantha Sandw., Kew Bull. 1949: 261 (1949). - Tipo: Guyana Inglesa, Eagle Mts, Fanshawe 1121. - Syn.: Psychotria aetantha (Sandw.) Steyerm., Mem. N. Y. Bot. Gard. 23: 665 (1972).

Palicourea fanshawei (Standl.) Borhidi, comb. nova, hoc loco - Basionym: Cephaëlis fanshawei Standl., Bull. Torrey Bot. Club 75: 574 (1948). - Tipo: Guyana Inglesa, Wallaba forest, savanna, Kaieteur Plateau, Maguire y Fanshawe 23296 (NY). - Syn.: Psychotria fanshawei (Standl.) Steyerm., Mem. N. Y. Bot. Gard. 23: 664 (1972).

Palicourea phaneroneura (Standl.) Borhidi, comb. nova, hoc loco - Basionym: Cephaëlis phaneroneura Standl., Publ. Field Columb. Mus., Bot. Ser. 22: 49 (1940), non Psychotria phaneroneura Standl., Publ. Field Columb. Mus., Bot. Ser. 7: 455 (1931). - Syn.: Psychotria peruviana Steyerm., Mem. N. Y. Bot. Gard. 23: 667 (1972). - Tipo: Perú, Loreto, Gamitanacocha, Río Mazán, Schunke 116.

Palicourea potaroensis (Sandw.) Borhidi, comb. nova, hoc loco - Basionym: Cephaëlis potaroensis Sandw. in Hook., Ic. Pl. 33, pl. 3300 (1935). - Tipo: Guyana Inglesa, Waratuk Portage, Potaro River, Tutin 470. - Syn.: Psychotria potaroensis (Sandw.) Steyerm., Mem. N. Y. Bot. Gard. 23: 662 (1972).

Palicourea psittacina (Steyerm.) Borhidi, comb. nova, hoc loco - Basionym: Psychotria psittacina Steyerm., Mem. N. Y. Bot. Gard. 23: 665 (1972). - Holotipo: 
Guyana Inglesa, Mt Ayanganna, Mazaruni River basin, Tillet, Tillet y Boyan 45007 (NY). - Ilustr.: Steyermark, Mem. N. Y. Bot. Gard. 23: 666 (1972).

Palicourea spectabilis (Steyerm.) Borhidi, comb. nova, hoc loco-Basionym: Psychotria spectabilis Steyerm., Mem. N. Y. Bot. Gard. 23: 664 (1972). - Tipo: Brazil, Pará, reg. Breves, fl. Aramá, Ducke 18828. - Nombre orig.: Cephaëlis duckei Standl., Field mus. Publ. Bot. 8: 374 (1931), non Palicourea duckei Standl., Publ. Field Columb. Mus., Bot. Ser. 22: 195 (1940).

\section{Sección 7. Neocephaëlis Borhidi, sectio nova}

Flores in capitulis compactis, monocephalis terminalibus plerumque pedunculatis, rariter subsessilibus dispositi, bracteis involucralibus extimis magnis et plerumque coloratis liberis vel plerumque basi connatis, flores individuales bracteolis duabus cincti. (17 species).

Typus sectionis: Palicourea tomentosa (Aubl.) Borhidi.

Palicourea apoda (Steyerm.) Delpr. et J. H. Kirkbr., J. Bot. Res. Inst. Texas 10: 412 (2016) - Basionym: Psychotria apoda Steyerm., Mem. N. Y. Bot. Gard. 23: 668 (1972). - Holotipo: Guyana Francesa, Territorio Oyac, Aublet (P). - Ilustr.: Steyermark, Fl. Venez. 3: 1587, fig. 277 (1974).

Palicourea bracteocardia (DC.) Delpr. et J. H. Kirkbr., J. Bot. Res. Inst. Texas 10: 414 (2016) - Basionym: Cephaëlis bracteocardia DC. Prodr. 4: 534 (1830). - Holotipo: Brazil, Bahia, Salzman (G-DC). - Syn.: Psychotria bracteocardia (DC.) Müll. Arg. in Mart., Fl. Bras. 6(5): 362 (1881); Psychotria ctenophora Steyerm., Mem. N. Y. Bot. Gard. 23: 690 (1972). - Ilustr.: Steyermark, Fl. Venez. 3: 1618, fig. 284 (1974).

Palicourea colorata (Willd. ex Roem. et Schult.) Borhidi, comb. nova, hoc loco - Basionym: Cephaëlis colorata Willd. ex Roem. et Schult., Syst. Veg. 5: 214 (1819). - Tipo: Brazil, Sieber (W-B). - Syn.: Psychotria colorata (Willd. ex Roem. et Scult.) Müll. Arg. in Mart., Fl. Bras. 6(5): 372 (1881); Psychotria calviflora Steyerm., Mem. N. Y. Bot. Gard. 23: 686 (1972).

Palicourea elata (Sw.) Borhidi, Acta Bot. Hung. 53: 244 (2011) - Basionym: Cephaëlis elata Sw., Prodr. p. 45 (1788). - Holotipo: Jamaica, Swartz s.n. (S!). - Syn.: Tapogomea elata (Sw.) Poir. in Lam., Encycl. Méth. Bot. 7: 585 (1806); Evea elata (Sw.) Standl., Contr. U. S. Natl. Herb. 18: 123 (1916); Psychotria elata (Sw.) Hammel in C. M. Taylor et al., Selbyana 12: 139 (1991). - Ilustr.: Burger y Taylor, Fieldiana Bot. n.s. 33: 31, t. 17 (1993).

Palicourea glomerulata (Donn.-Sm.) Borhidi, Acta Bot. Hung. 53: 245 (2011) - Basionym: Cephaëlis glomerulata Donn.-Sm., Bot. Gaz. (Crawforsville) 16: 12, t. 1 (1891). - Holotipo: Guatemala, Donnell-Smith 1637 (US). - Syn.: Psychotria glomerulata (Donn.-Sm.) Steyerm., Mem. N. Y. Bot. Gard. 23: 670 (1972). 
- Ilustr.: Burger y Taylor, Fieldiana Bot. n.s. 33: 32, t. 18 (1993); Borhidi, Rub. Mex. 2. ed. p. 386, fig. 86 (2012).

Palicourea hyalina (Steyerm.) Borhidi, comb. nova, hoc loco - Basionym: Psychotria hyalina Steyerm., Mem. N. Y. Bot. Gard. 23: 670 (1972). - Holotipo: Venezuela, Carabobo, Río San Gián, Julián y Cora Steyermark 95246 (VEN). Ilustr.: Steyermark, Fl. Venez. 3: 1590, fig. 278 (1974).

Palicourea muscosa (Jacq.) Borhidi, Acta Bot. Hung. 59: 18 (2017) - Basionym: Morinda muscosa Jacq., Stirp. Amer. p. 65, pl. 45 (1763). - Tipo: Martinique, Jacquin. - Syn.: Cephaëlis muscosa (Jacq.) Sw., Prodr. p. 46 (1788); Psychotria muscosa Steyerm., Mem. N. Y. Bot. Gard. 23: 671 (1972). - Ilustr.: Steyermark, Fl. Venez. 3: 1594, fig. 279 (1974).

Palicourea muscosa subsp. breviloba (Steyerm.) Borhidi, Acta Bot. Hung. 59: 19 (2017) - Basionym: Psychotria muscosa subsp. breviloba Steyerm., Mem. N. Y. Bot. Gard. 23: 672 (1972). - Holotipo: Trinidad, Alturas de Aripo, Broadway 9823 (NY).

Palicourea oblonga (DC.) Borhidi, comb. nova, hoc loco - Basionym: Cephaëlis oblonga DC., Prodr. 4: 535 (1830). - Holotipo: Guyana Francesa (GDC). - Syn.: Psychotria oblonga (DC.) Steyerm., Mem. N. Y. Bot. Gard. 23: 673 (1972).

Palicourea parimensis (Styerm.) Borhidi, comb. nova, hoc loco - Basionym: Psychotria parimensis Steyerm., Mem. N. Y. Bot. Gard. 23: 691 (1972). - Holotipo: Venezuela, Amazonas, Sierra Parima, Rio Siapa, Puig 1333 (VEN).

Palicourea rosacea (Steyerm.) Borhidi, comb. nova, hoc loco - Basionym: Psychotria rosacea Steyerm., Mem. N. Y. Bot. Gard. 23: 701 (1972).

Palicourea richardiana (Urb.) Borhidi et Oviedo, Acta Bot. Hung. 57: 276 (2015) - Basionym: Psychotria richardiana Urb., Symb. Ant. 7: 472 (1913). - Holotipo: Cuba, Pinar del Rio, Vuelta de Abajo, Valenzuela 321 (P!). - Syn.: Psychotria involucrata A. Rich. in Sagra Hist. Fis. Pol. Nat. Cub. 11: 27 (1850), non Sw. (1788); Palicourea cyanea Urb., Symb. Ant. 9: 168 (1923).

Palicourea rosea (Benth.) Borhidi, comb. nova, hoc loco - Basionym: Cephaëlis rosea Benth. in Hook., Journ. Bot. 3: 224 (1841). - Syn.: Psychotria rosea (Benth.) Müll. Arg. in Mart., Fl. Bras. 6(5): 360 (1881). - Tipo: Guyana Inglesa 1837, Essequibo, Schomburgk 156 (P). - Ilustr.: Steyermark, Fl. Venez. 3: 1614, fig. 283 (1974).

Palicourea rosea var. trichophora (Steyerm.) Borhidi, comb. nova, hoc loco - Basionym: Psychotria rosea var. trichophora Steyerm., Mem. N. Y. Bot. Gard. 23: 688 (1972). - Holotipo: Colombia, Sur de Santander, Valley of Dorado Creek, Haught 2190 (NY).

Palicourea ruelliifolia (Cham. et Schltdl.) Borhidi, comb. nova, hoc loco Basionym: Cephaëlis ruelliifolia Cham. et Schltdl., Linnaea 4: 134 (1829). - Tipo: 
South-Brazil, Sello. - Syn.: Psychotria ruelliifolia (Cham. et Schltdl.) Müll. Arg. in C. F. P. von Martius et auct. suc. (eds), Fl. Bras. 6(5): 364, pl. 50, fig. 1 (1881).

Palicourea spadicea (Pittier) Borhidi, comb. nova, hoc loco - Basionym: Pagamea spadicea Pittier, Bol. Soc. Venez. Cienc. Nat. 9: 123 (1944). - Tipo: Venezuela, Bolivar, Cerro Tonoro, Alto Paragua, Puig 847. - Syn.: Psychotria spadicea (Pittier) Standl. et Steyerm., Fieldiana Bot. 28: 1105 (1957). - Ilustr.: Steyermark, Fl. Venez. 3: 1596, fig. 280 (1974).

Palicourea tomentosa (Aubl.) Borhidi, Acta Bot. Hung. 53: 248 (2011) Basionym: Tapogomea tomentosa Aubl., Hist. Pl. Guian. 1: 160 (1775). - Holotipo: Guyana Francesa, Aublet s.n. (P). - Syn.: Cephaëlis tomentosa (Aubl.) Vahl, Eclog. Amer. 1: 19 (1796); Evea tomentosa (Aubl.) Standl., Contr. U. S. Natl. Herb. 1: 123 (1916); Cephaëlis hirsuta Mart. et Gal., Bull. Acad. Roy. Sci. Brux. 11: 35 (1844); Psychotria hirsuta (Mart. et Gal.) Müll. Arg. in Martius, Fl. Bras. 6(5): 370 (1881); Psychotria poeppigiana Müll. Arg. in Martius, Fl. Bras. 6(5): 370 (1881). - Ilustr.: Steyermark, Fl. Venez. 3: 1603, fig. 282 (1974); Borhidi Rub. Mex. 2. ed. p. 402, fig. 90 (2012).

Palicourea turbinella (Müll. Arg.) Borhidi, comb. nova, hoc loco - Basionym: Psychotria turbinella Müll. Arg. in Mart., Fl. Bras. 6(5): 374 (1881). - Tipo: Brazil, Amazonas, Rio Negro entre Barra y Barcellos, Spruce 1884. - Ilustr.: Steyermark, Fl. Venez. 3: 1599, fig. 281 (1974).

Sección 8. Axillares (Benth. et Hook.) Borhidi, comb. nova et status novus sub Palicourea Aubl.

Basionym: Cephaëlis Sw. series Axillares Benth. et Hook., Gen. Pl. 2: 128 (1873). - Syn.: Sectio Cephaëlis (Sw.) Müll. Arg. series Axillares (Benth. et Hook.) Steyerm., Mem. N. Y. Bot. Gard. 23: 693 (1972) sub Psychotria L.

Inflorescentiae densiflorae capitatae axillares, sessiles vel subsessiles, saepe bracteis stipularibus cinctae, flores individuales bracteolis duabus navicularibus vel cucullatis cincti. (11 species).

Typus sectionis Palicourea axillaris (Aubl.) Borhidi.

Palicourea anisopoda (Standl.) Borhidi, comb. nova, hoc loco - Basionym: Cephaëlis anisopoda Standl., Field Mus. Publ. Bot. 7: 424 (1931). - Tipo: Venezuela, Mérida, Tabay, Gehriger 380. - Syn.: Psychotria anisopoda (Standl.) Steyerm., Mem. N. Y. Bot. Gard. 23: 692 (1972).

Palicourea axillaris (Sw.) Borhidi, Acta Bot. Hung. 53: 243 (2011) - Basionym: Cephaëlis axillaris Sw., Prodr. Veg. Ind. Occ. 3: 45 (1788). - Holotipo: San Cristóbal, Swartz s.n. (S!). - Syn.: Tapogomea axillaris (Sw.) Poir. in Lam., Encycl. Méth. Bot. 7: 587 (1806); Evea axillaris (Sw.) Standl., Contr. U. S. Natl. 
Herb. 23: 1392 (1926); Psychotria aubletiana Steyerm., Mem. N. Y. Bot. Gard. 23: 694 (1972). - Ilustr.: Steyermark, Fl. Venez. 3: 1633, fig. 286 (1974).

Palicourea blepharophora (Standl.) Borhidi, comb. nova, hoc loco - Basionym: Cephaëlis blepharophora Standl., Field Mus. Publ. Bot. 7: 425 (1931). Tipo: Venezuela, Mérida, Tabay, Gehriger 387. - Syn.: Psychotria blepharophora (Standl.) Steyerm., Mem. N. Y. Bot. Gard. 23: 693 (1972).

Palicourea botryocephala (Standl.) Borhidi, comb. nova, hoc loco - Basionym: Cephaëlis botryocephala Standl., Field Mus. Publ. Bot. 8: 63 (1930). - Tipo: Venezuela, Estado Aragua, El Portachuelo, Pittier 10133. - Syn.: Psychotria botryocephala (Standl.) Steyerm., Mem. N. Y. Bot. Gard. 23: 692 (1972). - Ilustr.: Steyermark, Fl. Venez. 3: 1625, fig. 285 (1974).

Palicourea campylopoda (Standl.) Delpr. et J. H. Kirkbr., J. Bot. Res. Inst. Texas 10: 415 (2016) - Basionym: Psychotria campylopoda Standl., Publ. Field Columb. Mus., Bot. Ser. 7: 437 (1931). - Holotipo: Venezuela, Amazonas, Mt. Duida, Tate 534.

Palicourea celiae (Steyerm.) Borhidi, comb. nova, hoc loco - Basionym: Psychotria celiae Steyerm., Mem. N. Y. Bot. Gard. 23: 699, 700, fig. 88 (1972). Ilustr.: Steyermark, Fl. Venez. 3: 1641, fig. 287 (1974).

Palicourea eciliata (Steyerm.) Borhidi, comb. nova, hoc loco - Basionym.: Psychotria eciliata Steyerm., Mem. N. Y. Bot. Gard. 23: 693 (1972). - Tipo: Venezuela, Lara, Palojosco, Los Aposentos sobre Humocaro Bajo, Steyermark 55256. - Syn.: Cephaëlis tinctoria Standl. et Steyerm., Fieldiana Bot. 28: 568 (1953), non Palicourea tinctoria Standl. et Steyerm.

Palicourea norae (Steyerm.) Borhidi comb. nova, hoc loco - Basionym: Psychotria norae Steyerm., Mem. N. Y. Bot. Gard. 23: 703 (1972). - Holotipo: Venezuela, Táchira, cabeeras del Rio Quinimari, Steyermark y Dunsterville 101030 (VEN).

Palicourea ruiz-teranii (Steyerm.) Borhidi, comb. nova, hoc loco - Basionym: Psychotria ruiz-teranii Steyerm., Mem. N. Y. Bot. Gard. 23: 702 (1972). - Holotipo: Venezuela, Mérida, Rangel, San Rafael de Mucuchies, Ruiz-Teran 3199 (VEN).

Palicourea schlimii (Standl.) Borhidi, comb. nova, hoc loco - Basionym: Psychotria schlimii Standl., Publ. Field Columb. Mus., Bot. Ser. 7: 113 (1930). Tipo: Colombia, Ocaña, Schlim 695.

Palicourea tayloriae Borhidi, nomen novum, hoc loco - Nombre reemplazado: Psychotria dunstervilleorum Steyerm., Mem. N. Y. Bot. Gard. 23: 702 (1972), non Palicourea dunstervilleorum Steyerm., Acta Bot. Venez. 6: 175 (1971 publ. 1972). Dedicado al honor de Charlotte M. Taylor la especialista sobresaliente de la familia Rubiaceae. 
Sección 9. Tricephalium (Müll. Arg.) C. M. Taylor, Novon 54: 63 (2015)

Foliis chartaceis vel subcoriaceis venis intervenialibus abunde obsitis atque venis tertiariis dense reticulatis, floribus sessilibus in catipulos parvos vel majores coalitis atque bracteis bene evolutis circumdatis, fructibus in statu immaturo luteis vel rubris, stipulis circum ramos breviter connatis vel laminaribus, bilobatis vel emarginatis, apice rotundatis vel rotundatis et denticulatis, rariter lateraliter erodatis. (36 species).

Typus sectionis: Palicourea triadica (Müll. Arg.) C. M. Taylor.

Palicourea arenosa C. M. Taylor, nomen novum, Novon 24: 68 (2015) - Nombre reemplazado: Cephaëlis cuatrecasasii Standl. et Steyerm., Acta Biol. Venez. 4: 12 (1964). - Syn.: Psychotria cuatrecasasii (Standl. et Steyerm.) C. M. Taylor, non Palicourea cuatrecasasii Standl. ex Steyerm., Acta Biol. Venez. 4: 69 (1964). - Holotipo: Colombia, Norte de Santander, Cordillera Oriental, región de Sarare, hoya del Rio Margua, entre Campohermoso y Río Negro. J. Cuatrecasas 12894 (US-1851070).

Palicourea boyanii (Steyerm.) C. M. Taylor, Novon 24: 68 (2015) - Basionym: Psychotria boyanii Steyerm., Mem. N. Y. Bot. Gard. 23: 659 (1972). Holotipo: Guyana Inglesa, Merumé Mts. Rio Portang, Stephen y Carolyn Tillet 43954 (NY-132615).

Palicourea cenepensis (C. M. Taylor) C. M. Taylor, Novon 24: 81 (2015) Basionym: Psychotria cenepensis C. M. Taylor, Novon 4: 175 (1994). - Holotipo: Peru, Amazonas, $1 \mathrm{~km}$ de La Poza, banda E de Rio Santiago, Huashikat 178 (MO-2921205).

Palicourea conephoroides (Rusby) C. M. Taylor, Novon 24: 68 (2015) Basionym: Cephaëlis conephoroides Rusby, Bull. New York Bot. Gard. 4: 372 (1907). - Syn.: Psychotria conephoroides (Rusby) C. M. Taylor, Novon 4: 304 (1994). - Tipo: Bolivia, M. Bang 2866, lectotipo (NY-131017).

Palicourea costanensis (Steyerm.) C. M. Taylor Novon 24: 69 (2015) - Basionym: Psychotria costanensis Steyerm., Mem. N. Y. Bot. Gard. 23: 657 (1972). - Ilustr.: Steyermark, Fl. Venez. 3: 1572, fig. 273 (1974). - Nombre original: Cephaëlis macrocarpa Standl., Publ. Field Columb. Mus., Bot. Ser. 8: 64 (1930), non Palicourea macrocarpa Kunth 1820. - Holotipo: Venezuela, Colonia Tovar. A. Fendler 1990 (GH).

Palicourea crassifolia (Standl.) C. M. Taylor, Novon 24: 70 (2015) - Basionym: Cephaëlis crassifolia Standl., Publ. Field Columb. Mus., Bot. Ser. 7: 77 (1930). - Syn.: Psychotria cordobensis C. M. Taylor, Novon 4: 304 (1994), non Psychotria crassifolia Miq. (1869). - Holotipo: Colombia, Valle de Cauca, Córdoba, E. P. Killip 5133 (US-1140556). 
Palicourea ernestii (K. Krause) C. M. Taylor, Novon 24: 70 (2015) - Basionym: Cephaëlis ernestii K. Krause, Notizbl. Bot. Gart. Berlin-Dahlem 6: 210 (1914). - Syn.: Psychotria transiens Wernham, J. Bot. 52: 314 (1914). - Holotipo: Brazil, Roraima-tepui, Ule 8771 (B十), lectotipo (K-432821).

Palicourea flaviflora (K. Krause) C. M. Taylor, Novon 24: 82 (2015) Basionym: Uragoga flaviflora K. Krause, Bot. Jahrb. Syst. 40: 344 (1908). - Syn.: Psychotria flaviflora (K. Krause) C. M. Taylor, Monogr. Syst. Bot. Missouri Bot. Gard. 45: 1258 (1993). - Holotipo: Peru, Huánuco, prov. Huamalies, inter Monzon et Huallaga, Weberbauer (B十), lectotipo (G). - Ilustr.: Novon 24: 79, fig. 3E-F (2015).

Palicourea grandiceps C. M. Taylor, Novon 24: 82 (2015) - Holotipo: Ecuador, Napo, cantón El Chaco, Río Granacillo, campamento de INECEL, "Codo Alto", W. Palacios 5488 (QCNE-39191). - Ilustr.: Novon 24: 75, fig. 2C-F (2015).

Palicourea heteroneura (Steyerm.) C. M. Taylor, Novon 24: 70 (2015) Basionym: Psychotria heteroneura Steyerm., Mem. N. Y. Bot. Gard. 23: 714, fig. 91 (1972). - Ilustr.: Steyermark, Fl. Venez. 3: 1678, fig. 296 (1974). - Holotipo: Brazil, Amazonas, from Igarapé Anta up slopes to Pico da Neblina, Río Negro, Río Cauaburi, Río Maturacá, Silva y Brazão 60607 (NY-132690).

Palicourea hypochlorina (C. M. Taylor) C. M. Taylor, Novon 24: 71 (2015) - Basionym: Psychotria hypochlorina C. M. Taylor, Sida 17: 713, fig. 2A-B (1997). - Holotipo: Peru, Loreto, prov. Maynas, Soboya, Río Pintuyacu, R. Vásquez et al. 7420 (MO-05079650).

Palicourea jenmani (Wernh.) C. M. Taylor, Novon 24: 71 (2015) - Basionym: Cephaëlis jenmani Wernham, Journ. Bot. 52: 323 (1914), non Psychotria jenmanii Urb., Symb. Ant. 7: 440 (1913). - Holotipo: Guyana Inglesa, Sheenabown, Potaro River, Jenman 1291 (K-174490). - Syn.: Psychotria mazaruniensis Standl., Bull. Torrey Bot. Club 67: 298 (1940).

Palicourea jervisei (Standl.) C. M. Taylor, Novon 24: 71 (2015) - Basionym: Cephaëlis jervisei Standl., Publ. Field Columb. Mus., Bot. Ser. 7: 79 (1930). - Syn.: Psychotria jervisei (Standl.) C. M. Taylor, Novon 4: 305 (1994). - Holotipo: Colombia, Antioquia, Jervis s.n. (K-432891).

Palicourea jungiana C. M. Taylor, Novon 24: 88 (2015) - Nombre reemplazado: Psychotria longipes Müll. Arg. in C. F. P. von Martius et auct. suc. (eds), Fl. Bras. 6(5): 354 (1881), non Palicourea longipes Rusby, Bull. New York Bot. Gard. 8: 121 (1912); Uragoga longipes (Müll. Arg.) Kuntze, Revis. Gen. Pl. 2: 961 (1891). - Lectotipo: Brazil, Rio de Janeiro, A. Glaziou 6037 (P).

Palicourea kaieteurensis (Wernh.) C. M. Taylor, Novon 24: 71 (2015) Basionym: Cephaëlis kaieteurensis Wernham, J. Bot. 52: 313 (1914). - Syn.: Psychotria appuniana Steyerm., Mem. N. Y. Bot. Gard. 23: 660 (1972), non Psychot- 
ria kaieteurensis Sandw. (1940). - Holotipo: Guyana Inglesa, Kaieteur Falls, C. F. Appun s.n. (BM).

Palicourea lewisiorum C. M. Taylor, Novon 24: 84 (2015) - Holotipo: Peru, Loreto, Puranchim, Rio Sinchinyacu, W. H. Lewis et al. 11879 (MO6240024). - Ilustr.: Novon 24: 79, fig. 3A-D (2015).

Palicourea neillii C. M. Taylor, Novon 24: 72 (2015) - Holotipo: Ecuador, Zamora-Chinchipe, Cantón Yantzaza, Cordillera del Cóndor, Rio Machinaza en los Encuentros, summit area Hollín, Neill y Kajekai 16980 (QCNE-0226883). - Ilustr.: Novon 24: 72, fig. 1C-F (2015).

Palicourea oleandrella (Standl.) C. M. Taylor, Novon 24: 86 (2015) - Basionym: Cephaëlis oleandrella Standl., Publ. Field Columb. Mus., Bot. Ser. 4: 334 (1929). - Syn.: Psychotria oleandrella (Standl.) C. M. Taylor, Monogr. Syst. Bot. Missouri Bot. Gard. 45: 1258 (1993). - Holotipo: Peru, Junin, mnt slope La Merced, Macbride 5635 (F-536673).

Palicourea ownbeyi (Standl. ex C. M. Taylor) C. M. Taylor, Novon 24: 86 (2015) - Basionym: Psychotria ownbeyi Standl. ex C. M. Taylor, Novon 4: 176 (1994). - Holotipo: Ecuador, Napo, Cantón Archidona, S slopes Volcán Sumaco near Rio Huateraco, D. Neill et al. 9164 (MO-4672678).

Palicourea oxapampana C. M. Taylor, Novon 24: 88 (2015) - Holotipo: Peru, Pasco, prov. Oxapampa, Villa Rica-Yesú road, D. N. Smith et al. 5454 (USM).

Palicourea paujilensis C. M. Taylor, Novon 24: 73 (2015) - Holotipo: Perú, Pasco prov. Oxapampa, distr. Palcazú, El Paujíl, H. van der Werff et al. 20023 (HOXA). - Ilustr.: Novon 24: 72, fig. 1A-B (2015).

Palicourea perinsignis C. M. Taylor, Novon 24: 86 (2015) - Nombre reemplazado: Mapouria insignis Müll. Arg. in C. F. P. von Martius et auct. suc. (eds), Fl. Bras. 6(5): 424 (1881), non Psychotria insignis Standl., Contr. U. S. Natl. Herb. 18: 130 (1916), nec Uragoga insignis (Müll. Arg.) Kuntze. - Holotipo: Brazil, Pará, prov. Para, Martius s.n. (G).

Palicourea pleiocephala (Müll. Arg.) C. M. Taylor, Novon 24: 89 (2015) - Basionym: Psychotria pleiocephala Müll. Arg., Flora 59: 552 (1876). - Syn.: Uragoga pleiocephala (Müll. Arg.) Kuntze, Revis. Gen. Pl. 2: 962 (1891). - Tipo: Brazil, Minas Gerais, Serra d'Ouroubranco, L. Riedel 2875, lectotipo (BR-531607).

Palicourea schraderoides (K. Krause) C. M. Taylor, Novon 24: 74 (2015) - Basionym: Uragoga schraderoides K. Krause, Bot. Jahrb. Syst. 40: 344 (1908). - Syn.: Psychotria schraderoides (K. Krause) C. M. Taylor, Monogr. Syst. Bot. Missouri Bot. Gard. 45: 1258 (1993). - Holotipo: Peru, Loreto, Moyohamba, Weberbauer 4750 (B十), lectotipo (MOL-00500).

Palicourea schunkei (C. M. Taylor) C. M. Taylor, Novon 24: 87 (2015) Basionym: Psychotria schunkei C. M. Taylor, Monogr. Syst. Bot. Missouri Bot. Gard. 45: 1258 (1993). - Holotipo: Perú, Killip y A. C. Smith 29303 (F-607497). - 
Nombre original: Cephaëlis killipii Standl., Publ. Field Columb. Mus., Bot. Ser. 8: 187 (1930), non Palicourea killipii Standl., Publ. Field Columb. Mus., Bot. Ser. 7: 136 (1930).

Palicourea sessilis (Vell.) C. M. Taylor, Novon 24: 90 (2015) - Basionym: Coffea sessilis Vell., Fl. Flumin.: 64 (1829), non Psychotria sessilis Vell. - Syn.: Psychotria vellosiana Benth., Linnaea 23: 464 (1850). - Tipo: Brazil, Campis apricis maritimis, J. M. da C. Velloso s.n. Fl. Flumin. Icon 2, tab. 20 (1827), lectotipo.

Palicourea simpsonii C. M. Taylor Novon 24: 75 (2015) - Holotipo: Colombia. Vaupés: Rio Pira, Paraná, mission San Miquel, Cañon Colorado, E. W. Davis 137 (COL-169-356). - Ilustr.: Novon 24: 75, fig. 2A-B (2015).

Palicourea sororopanensis (Standl. et Steyerm.) Borhidi, comb. nova, hoc loco - Basionym: Psychotria sororopanensis Standl. et Steyerm., Fieldiana Bot. 28: 605 (1953). - Holotipo: Venezuela, Bolívar, Sororopán-tepui, J. A. Steyermark 60175 (F-1182123). - Syn: Palicourea sessilis subsp. sororopanensis (Standl. et Steyerm.) C. M. Taylor Novon 24: 92 (2015).

Palicourea speluncae (Standl. et Steyerm.) C. M. Taylor, Novon 24: 76 (2015) - Basionym: Psychotria speluncae Standl. et Steyerm., Fieldiana Bot. 28: 606 (1953). - Holotipo: Venezuela, Bolivar, Ptari-tepui, Steyermark 59521 (F1182101).

Palicourea tepuiensis (Steyerm.) C. M. Taylor, Novon 24: 77 (2015) - Basionym: Cephälis tepuiensis Steyerm., Mem. N. Y. Bot. Gard. 17: 430 (1967). Holotipo: Venezuela, Bolívar, alto Río Cuyuni, Río Uiri-Yuk, cumbre La Escalera, Maguire y J. A. Steyermark 46864 (VEN-63024). - Syn.: Psychotria tepuiensis (Steyerm.) Steyerm., Mem. N. Y. Bot. Gard. 23: 661 (1972). - Ilustr.: Steyermark, Fl. Venez. 3: 1580, fig. 275 (1974).

Palicourea triadica (Müll. Arg.) C. M. Taylor, Novon 24: 77 (2015) - Basionym: Mapouria triadica Müll. Arg. in C. F. P. von Martius et auct. suc. (eds), Fl. Bras. 6(5): 423 (1881). - Syn.: Uragoga triadica (Müll. Arg.) Kuntze, Revis. Gen. Pl. 2: 963 (1891). - Holotipo: Brazil, Amazonas, regione inferiore Río Negro ad Porto dos Miranhas, Martius s.n. (M).

Palicourea tricephala (Müll. Arg.) C. M. Taylor, Novon, 24: 77 (2015) Basionym: Mapouria tricephala Müll. Arg. in C. F. P.von Martius et auct. suc. (eds), Fl. Bras. 6(5): 423 (1881). - Syn.: Psychotria tricephala (Müll. Arg.) Zappi, Prodr. Fl. Matogross., Ser. B3: 259 (1998). - Holotipo: Brazil, Amazonas, Rio Negro, Uapés, Spruce 2666 (G).

Palicourea ucayalina C. M. Taylor, Novon 24: 78 (2015) - Holotipo: Perú, Ucayali, prov. Padre Abad. Fundu Yacu Mama carr. Aguaytia-Boquerón, Schunke Vigo y Graham 15678 (MOL). - Ilustr.: Novon 24: 79, fig. 3G-L.

Palicourea valenzuelana C. M. Taylor, Novon 24: 80 (2015) - Holotipo: Perú, Pasco, Oxapampa, distr. Pozuzo, sector Algo Lagarto, Villa Progreso, 
Res. Comunal Yanesha, L. Valenzuela et al. 15833 (USM). - Ilustr.: Novon 24: 72, fig. 1G-H (2015).

Palicourea vareschii (Steyerm.) Borhidi, comb. nova, hoc loco - Basionym: Psychotria vareschii Steyerm., Mem. N. Y. Bot. Gard. 23: 706, fig. 89 (1972). Holotipo: Venezuela, Amazonas, Casiquiare, Caño Monomi, Vareschi 7790 (VEN). - Ilustr.: Steyermark, Fl. Venez. 3: 1662, fig. 291 (1974).

Palicourea yneziae C. M. Taylor, Novon 24: 81 (2015) - Nombre reemplazado: Rudgea mexiae Standl., Publ. Field Columb. Mus., Bot. Ser. 13(6): 165 (1936), non Palicourea mexiae Standl. (1940). - Holotipo: Perú, Loreto, above Pongo de Manseriche, mouth of Rio Santiago. Ynez Mexia 6366 (F-718409).

Palicourea zevallosii (C. M. Taylor) C. M. Taylor, Novon 24: 87 (2015) Basionym: Psychotria zevallosii C. M. Taylor, Monogr. Syst. Bot. Missouri Bot. Gard. 45: 1258 (1993). - Nombre reemplazado: Cephaëlis williamsii Standl., Publ. Field Columb. Mus., Bot. Ser. 8: 185 (1930), non Palicourea williamsii Rusby (1920), nec Psychotria williamsii Standl. (1930). - Holotipo: Peru, Loreto, Manfinfa upper Rio Nanay, I. J. Williams 1114 (F-604625).

\section{Sección 10. Cordifoliae Borhidi, sectio nova}

Foliis oppositis sessilibus vel subsessilibus coriaceis vel chartaceis, basi cordatis, inflorescentiae terminales paniculatae, floribus laxe dispositis vel in capitulis binatis vel trifloris coaliti. (3 species).

Typus sectionis: Palicourea cardiomorpha (C. M. Taylor) Borhidi.

Palicourea cardiomorpha (C. M. Taylor) Borhidi, comb. nova, hoc loco - Basionym: Psychotria cardiomorpha C. M. Taylor et A. Pool, Novon 4: 303 (1994). - Holotipo: Venezuela, Amazonas, San Balthazar fl. Atapabo, Humboldt y Bonpland (P). - Syn.: Psychotria cordifolia Kunth in F. W. H. von Humboldt, A. J. A. Bonpland et C. S. Kunth, Nov. Gen. Sp. 3: 365 (1819), nom. illeg. - Ilustr.: Steyermark, Fl. Venez. 3: 1664, fig. 292 (1974).

Palicourea cardiomorpha subsp. perpusilla (Steyerm.) Borhidi, comb. nova, hoc loco - Basionym: Psychotria cordifolia subsp. perpusilla Steyerm., Mem. N. Y. Bot. Gard. 23: 708 (1972). - Holotipo: Guyana Inglesa, Pirara etc. 184142, Schomburgk 292 (P).

Palicourea sipapoensis (Steyerm.) Borhidi, comb. nova, hoc loco - Basionym: Psychotria sipapoensis Steyerm., Mem. N. Y. Bot Gard. 23: 708, fig. 90 (1972). - Holotipo: Venezuela, Amazonas, Cerro Sipapo, Maguire y Politi 28778 (NY). - Ilustr.: Steyermark, Fl. Venez. 3: 1667, fig. 293 (1974). 
Sección 11. Microphyllae Borhidi, sectio nova sub Palicourea Aubl. subgen. Heteropsychotria Steyerm. emend. Borhidi

Frutices vel subfrutices parvifoliati, foliis usque ad $5 \mathrm{~cm}$ longis, plerumque coriaceis vel chartaceis, paucinervibus et venis inconspicuis vel obsoletis suffultis, inflorescentae terminales vel subterminales, plerumque subsessiles, pauciradiatae et pauciflorae, foliis superioribus breviores, cymoso-corymbosae, floribus plerumque solitariis, sessilibus, unibracteolatis. (7 species).

Typus sectionis: Palicourea orientensis Borhidi et Oviedo.

Palicourea ekmanii (Urb.) Borhidi et Oviedo, Acta Bot. Hung. 58: 6 (2016) - Basionym: Psychotria ekmanii Urb., Symb. Ant. 9: 167 (1923). - Holotipo: Cuba, Sierra Maestra, La Bayamesa, Ekman 7152 (B十), lectotipo (S!).

Palicourea micrantha Urb. et Ekm., Ark. Bot. 22A (10): 97 (1929) - Holotipo: Santo Domingo; Azua prope Las Lagunas, Ekman H 6410 (S!). - Syn.: Psychotria azuensis Alain, Moscosoa 8: 10 (1994), nom. superfl.

Palicourea moralesii (Acuña et Roig) Borhidi, Acta Bot. Hung. 51: 276 (2009) - Basionym: Psychotria moralesii Acuña et Roig, Brittonia 14: 224 (1962). - Tipo: Cuba, Moa.

Palicourea odorata (Wr. ex Griseb.) Borhidi et Oviedo, Acta Bot. Hung. 58: 7 (2016) - Basionym: Psychotria odorata Wr. ex Griseb., Cat. Pl. Cub. p. 138 (1866). - Tipo: Cuba, Oriente, Mayari abajo, Ch. Wright 2752. - Holotipo: GOET, isotipos: F, GH, US, HAC!.

Palicourea orientensis Borhidi et Oviedo, Acta Bot. Hung. 57: 276 (2015) - Nombre reemplazado: Psychotria subalata Wr. ex Griseb. Cat. Pl. Cub. p. 137 (1866), non Palicourea subalata Standl. et Steyerm. (1964). - Tipo: Cuba oriental, Mayari, Ch. Wright 2754. - Holotipo: GOET, isotipo: GH, HAC!.

Palicourea toensis (Britt. et Wils.) Standl., Field Mus. Publ. Bot. 11: 231 (1936) - Basionym: Psychotria toensis Britt. et Wils., Mem. Torrey Bot. Club 16: 113 (1920). - Holotipo: Cuba, Campo Toa, Rio Yamanigüey, Shafer 4009 (NY). - Syn.: Palicourea purpurascens Urb., Symb. Ant. 9: 167 (1923).

Palicourea torbeciana Urb. et Ekm., Ark. Bot. 21A(5): 87 (1927) - Holotipo: Haiti; Massif de la Hotte, Torbec, La Mare Proux, Ekman H 5270 (S!). Syn.: Psychotria torbeciana (Ekm. et Urb.) Alain, Moscosoa 8: 10 (1994).

\section{LITERATURA CONSULTADA}

Andersson, L. (2001): Margaritopsis (Rubiaceae, Psychotrieae) is a pantropical genus. Syst. Geogr. Pl. 71: 73-85. https://doi.org/10.2307/3668754

Andersson, L. (2002a): Relationships and generic circumscriptions in the Psychotria complex (Rubiaceae, Psychotrieae). - Syst. Geogr. Pl. 72: 167-202. https://doi.org/ $10.2307 / 3668767$ 
Andersson, L. (2002b): Re-establishment of Carapichea (Rubiaceae, Psychotrieae). - Kew Bull. 57: 363-374. https://doi.org/10.2307/4111112

Andersson, L. y Rova, J. H. E. (1999): The rps16 intron and the phylogeny of the Rubiaceae. - Plant Syst. Evol. 214: 161-186. https://doi.org/10.1007/BF00985737

Borhidi, A. (2011): Transfer of the Mexican species of Psychotria subgen. Heteropsychotria to Palicourea based on morphological and molecular evidences. - Acta Bot. Hung. 53: 241-250. https://doi.org/10.1556/ABot.53.2011.3-4.4

Borhidi, A. (2012): Rubiáceas de México. Segunda y ampliada edición. - Akadémiai Kiadó, Budapest, 610 pp.

Borhidi, A. L. (2015): Some nomenclatural corrections in the Caribbean flora. - Acta Bot. Hung. 57: 279-281. https://doi.org/10.1556/034.57.2015.3-4.3

Borhidi, A. L. (2017): El subgénero Heteropsychotria (Rubiaceae, Palicoureeae) en México y Mesoamerica. Acta Bot. Hung. 59: 13-23. https://doi.org/10.1556/034.59.2017.1-2.3

Borhidi, A., Martínez-Salas, E. y Ramos Álvarez, C. H. (2015): An uncommon pyrene type and two new genera in the neotropical Psychotrieae (Rubiaceae). - Acta Bot. Hung. 57: 241-270. https://doi.org/10.1556/034.57.2015.3-4.1

Borhidi, A. y Oviedo-Prieto, R. (2015): Correcciones a la taxonomía y nomenclatura del género Psychotria s. l. en las floras de Cuba, La Española y Jamaica. - Acta Bot. Hung. 57: 271-278. https://doi.org/10.1556/034.57.2015.3-4.2

Borhidi, A. L., Oviedo-Prieto, R. y Fernández-Zequeira, M. (2016): Nuevos resultados de la revisión taxonómica de los géneros Palicourea y Psychotria (Rubiaceae, Psychotrieae) en Cuba. - Acta Bot. Hung. 58: 1-48. https://doi.org/10.1556/034.58.2016.1-2.1

Bremer, B. (2009): A review of molecular phylogenetic studies of Rubiaceae. - Ann. Mo. Bot. Gard. 96: 4-26. https://doi.org/10.3417/2006197

Burger, W. C. y Taylor, C. M. (1993): Family 202. Rubiaceae. In: Burger, W. C. (ed.): Flora Costaricensis. - Fieldiana, Bot. n.s. 33: 1-333.

Clausen, J., Keck, D. D. y Hiesey, W. M. (1948): Experimental studies on the nature of species. III: Environmental responses of climatic races of Achillea. - Carnegie Institution of Washington, Publication 581, Washington, D.C.

Delprete, P. and Kirkbride, H. J. (2016): New combinations and new names in Palicourea (Rubiaceae) for species of Psychotria subgenus Heteropsychotria occurring in the Guianas. - J. Bot. Res. Inst. Texas 10: 409-416.

Dwyer, D. J. (1980a): Rubiaceae. In: Woodson, R. E. Jr., Scheryl, R. W. et al. (eds): Flora of Panama. Part 1. - Ann. Mo. Bot. Gard. 67(1): 1-256. https://doi.org/10.2307/2398954

Dwyer, D. J. (1980b): Rubiaceae. In: Woodson, R. E. Jr., Scheryl, R. W. et al. (eds): Flora of Panama. Part IX. Family 179. Rubiaceae. Part II. - Ann. Mo. Bot. Gard. 67(2): 257-522. https://doi.org/10.2307/2398901

Govaerts, R. (2015): World checklist of Rubiaceae. - Royal Botanic Gardens, Kew. http://apps. kew.org/wcsp

Nepokroeff, M., Bremer, B. y Sytsma, K. J. (1999): Reorganization of the genus Psychotria and tribe Psychotrieae (Rubiaceae) inferred from ITS and rbcL sequence data. - Syst. Bot. 24(1): 5-27. https://doi.org/10.2307/2419383

Petit, E. (1964): Les espèces africaines du genre Psychotria L. Rubiaceae. I. - Bull. Rijksplantentuin (Brussel) 34: 1-228. https://doi.org/10.2307/3667213

Piesschaert, F., Robbrecht, E. y Smets, E. (2001): Patterns in pyrenes: the systematic significance of pyrene morphology in Chassalia (Rubiaceae, Psychotrieae) and related genera. - Flora 196: 121-131. 
Razafimandimbison, S. G., Taylor, C. M., Wikström, N., Pailler, T., Khodabandeh, A. y Bremer, B. (2014): Phylogeny and generic limits in the sister tribes Psychotrieae and Palicoureeae (Rubiaceae): evolution of schizocarps in Psychotria and origin of bacterial leaf-nodules of the Malgasy species. - Amer. J. Bot. 101(7): 1102-1126. http:// dx.doi.org/10.3732/ajb.1400076

Robbrecht, E. (1988): Tropical woody Rubiaceae. Characteristic features and progressions. Contributions to a new subfamilial classification. - Opera Bot. Belg. 1: 1-271.

Robbrecht, E. (1989a): Germination slits in Rubiaceae diaspores. - Progr. y Abstr., Flora Males. Symp., Leiden, August, 1989, p. 53.

Robbrecht, E. (1989b): A remarkable new Chazaliella (African Psychotrieae), exemplifying the taxonomic value of pyrene characters in the Rubiaceae. - Bull. Mus. natn. Hist. Nat. Paris, sér. 4, 11: 341-345.

Ruiz Lopez, H. y Pavón, J. (1794): Florae Peruvianae et Chilensis Prodromus. - La Imprenta de Sancha, Madrid.

Schnell, R. (1960): Note sur le genre Cephaëlis et le problème de l'évolution parallèle chez les Rubiacées. - Bull. Rijksplantentuin (Brussel) 30:357-373. https://doi.org/10.2307/3667307

Standley, P. C. (1918-1934): Rubiaceae. North American Flora 32: 1-300. Standley, P. C. (1926): Rubiaceae. In: Trees and shrubs of Mexico. - Contr. U. S. Natl. Herb. 23: 13491394.

Standley, P. C. (1928): New plants from Central America XI. - J. Wash. Acad. Sci. 18: 160-169.

Standley, P. C. (1930): Studies on American plants IV. - Publ. Field Mus. Nat. Hist., Bot. Ser. 8: $133-236$.

Standley, P. C. (1938): Rubiaceae. In: Flora of Costa Rica. - Publ. Field Mus. Nat. Hist., Bot. Ser. 18: $1264-1380$.

Standley, P. C. (1940a): Studies on American plants X. - Publ. Field Mus. Nat. Hist., Bot. Ser. 22: 101-132.

Standley, P. C. (1940b): Studies on American plants XI. - Publ. Field Mus. Nat. Hist., Bot. Ser. 22: $180-218$.

Standley, P. C. y Steyermark, J. A. (1940a): Studies on Central American plants I. - Publ. Field Mus. Nat. Hist., Bot. Ser. 22: 219-322.

Standley, P. C. y Steyermark, J. A. (1940b): Studies on Central American plants II. - Publ. Field Mus. Nat. Hist., Bot. Ser. 22: 358-387.

Standley, P. C. y Steyermark, J. A. (1947): Studies on Central American plants III. - Publ. Field Mus. Nat. Hist., Bot. Ser. 23: 3-28.

Steyermark, J. A. (1972): Rubiaceae. In: Steyermark, J. A., Maguire, B., Wurdack, J. J. et al.: Botany of the Guyana Highland, Part IX. - Mém. New York Bot. Gard. 23: 227-832.

Steyermark, J. A. (1974): Rubiaceae. - In: Lasser, T. y Steyermark, J. A. (eds): Flora de Venezuela. Inst. Bot., Caracas, Vol. 9(1-3), 2070 pp.

Taylor, C. M. (1989): Revision of Palicourea in Mexico and Central America. - Syst. Bot. Monogr. 26: 1-102. https://doi.org/10.2307/25027720

Taylor, C. M. (2001): Overview of the neotropical genus Notopleura (Rubiaceae, Psychotrieae) with the description of some new species. - Ann. Mo. Bot. Gard. 88: 478-515. https://doi.org/10.2307/3298587

Taylor, C. M. (2004): Rubiacearum Americanarum Magna Hama Pars XVI. New species, a new subspecies, and an overlooked species of Psychotria subg. Heteropsychotria from Mexico, Central America, and western South America. - Novon 14(4): 493-508.

Taylor, C. M. (2005): Margaritopsis (Rubiaceae, Psychotrieae) in the Neotropics. - Syst. Geogr. Pl. 75(2): 161-177. https://doi.org/10.2307/3668574 
Taylor, C. M. (2012a): Palicourea Aubl. - In: Davidse, G., Sousa, M., Knapp, S. y Chiang, F. (eds): Flora Mesoamericana, Vol. 4/2, pp. 163-181.

Taylor, C. M. (2012b): Psychotria L. - In: Davidse, G., Sousa, M., Knapp, S. y Chiang, F. (eds): Flora Mesoamericana, Vol. 4/2, pp. 192-241.

Taylor, C. M. (2015a): Rubiacearum Americanarum Magna Hama Pars XXXIII. The new group Palicoureae sect. Didymocarpae with four new species and two new subspecies (Palicoureeae). - Novon 23(4): 452-478. https://doi.org/10.3417/2012003

Taylor, C. M. (2015b): Rubiacearum Americanarum Magna Hama Pars XXXIV. The new group Palicourea sect. Tricephalium with eight new species and a new subspecies (Palicoureeae). - Novon 24(1) 55-95. https://doi.org/10.3417/2015001

Taylor, C. M. y Gereau, R. E. (2013): The genus Carapichea (Rubiaceae, Psychotrieae). Ann. Mo. Bot. Gard. 99: 100-127. https://doi.org/10.3417/2011064

Taylor, C. M. y Hollowell, V. C. (2016): Rubiacearum Americanarum Magna Hama Pars XXXV. The new group Palicourea sect. Nonatelia with five new species (Palicoureeae). - Novon 25: 69-110. https://doi.org/10.3417/2015012

Taylor, C. M. y Lorence, D. H. (1992): Notes on Psychotria subgenus Heteropsychotria (Rubiaceae: Psychotrieae) in Mexico and Northern Central America. - Novon 2: 259-266. https://doi.org/10.2307/3391564

Taylor, C. M., Bruniera, C. y Zappi, D. (2015): Taxonomic transfers in Neotropical Psychotrieae: New combinations in Rudgea and Palicourea. - Kew Bull. 70(45): 1-7.

Taylor, C. M., Lorence, D. H. y Gereau, R. E. (2010): Rubiacearum Magna Hama Pars XXV: The nocturnally flowering Psychotria domingensis-Coussarea hondensis group plus three other Mesoamerican Psychotria transfer to Palicourea. - Novon 20(4): 481-492. https://doi.org/10.3417/2009124

Taylor, C. M., Steyermark, J. A., Delprete, P., Persson, C., Costa, C., Vicentini, A. y Cortés, R. (2004): Rubiaceae. - In: Berry, P., Yatskievych, K. y Holst, B. K. (eds): Flora of the Venezuelan Guayana. Vol. 8. Missouri Bot. Gard. Press, St. Louis, pp. 497-847.

Verdcourt, B. (1976): Rubiaceae (part 1). - In: Polhill, R. M. (ed.): Flora of tropical East Africa. Agents for Overseas Governments and Administration, London, $414 \mathrm{pp}$.

WCSP (2014): World checklist of selected plant families. Facilitated by the Royal Botanic Gardens, Kew. http://apps.kew.org./wcsp/, accessed 6 December, 2014. 